\title{
Plane-stress crack-tip fields for power-law hardening orthotropic materials
}

\author{
JWO PAN ${ }^{1}$ and C. FONG SHIH ${ }^{2}$ \\ ${ }^{1}$ Department of Mechanical Engineering and Applied Mechanics, The University of Michigan, Ann Arbor, \\ MI 48109, USA; ${ }^{2}$ Division of Engineering, Brown University, Providence, RI 02912, USA
}

Received 9 September 1987; accepted in revised form 11 January 1988

\begin{abstract}
Plane stress mode I near-tip fields in orthotropic materials are examined. Plastic orthotropy is described by Hill's quadratic yield function and the strain hardening behavior is given by an appropriate generalization of a uniaxial tensile power-law stress-strain relation. Pronounced changes in the pattern of the angular variations of crack-tip fields have been observed with the degree of plastic orthotropy and the amount of strain hardening. Possible shapes and sizes of plastic zones (as inferred from effective stress contours) are presented for high- and low-hardening materials and a wide range of plastic orthotropy. The shape of the plastic zone for a particular case of plastic orthotropy agreed remarkably well with the zone of intense straining induced by an appropriately orientated crack within a graphite/epoxy laminate.
\end{abstract}

\section{Introduction}

Anisotropic plastic behavior in polycrystals arises from preferred crystallographic orientations for slip and twinning and from the development of texture caused by large plastic deformation. To describe the plastic anisotropic behavior, Hill [1] proposed a phenomenological quadratic yield function for orthotropic materials. Bassani [2], using the Bishop-Hill procedure [3], constructed a family of yield functions for polycrystals with a wide range of transversely isotropic properties. Later, Hill [4] proposed a non-quadratic yield function to account for the so-called anomalous behaviour of certain materials. To gain some understanding of the plastic flow near the tip of a crack in an orthotropic material, we adopted the simple Hill quadratic yield function and the normality flow rule to model the plastic flow behavior.

The asymptotic near-tip solutions for a crack in both power-law hardening and perfectly plastic isotropic materials have been presented by Hutchinson $[5,6]$ and Rice and Rosengren [7]. Shih $[8,9]$ obtained the asymptotic near-tip solutions for both power-law hardening and perfectly plastic isotropic materials under combined mode I and II conditions. Hayashi [10] determined the asymptotic near-tip solutions for some power-law hardening materials with mild orthotropy. Pan and Shih [11] and Pan [12] carried out a systematic study of the asymptotic near-tip solutions for both power-law hardening and perfectly plastic orthotropic materials under plane-strain conditions.

Here, we present mode I plane-stress crack-tip solutions for power-law hardening orthotropic materials to show the effects of strain hardening and plastic orthotropy on the crack-tip fields under plane-stress conditions. Furthermore, the low-hardening powerlaw crack-tip solutions are used as a guide to construct the corresponding perfectly plastic crack-tip solutions [18]. An excellent discussion of plastic crack-tip fields in 
homogeneous materials and their relevance to the $J$-based [13] phenomenological nonlinear fracture mechanics can be found in the review paper by Hutchinson [14].

\section{Orthotropic plasticity}

We adopt the small-strain deformation plasticity approach to examine the effects of plastic orthotropy and plastic hardening on crack-tip fields. We consider cases where the pronounced plastic orthotropy is already firmly established and restrict the discussion to a range of plastic straining such that further change of plastic orthotropy is negligible. The axes of a Cartesian coordinate system, $x_{1}, x_{2}$, and $x_{3}$, are chosen to coincide with the axes of the orthotropic symmetry of a material, $X_{1}, X_{2}$, and $X_{3}$. We adopt the Hill quadratic yield condition [1] for the orthotropic material. The yield condition can be expressed in the form

$$
\begin{aligned}
\phi\left(\sigma_{i j}\right)= & F\left(\sigma_{22}-\sigma_{33}\right)^{2}+G\left(\sigma_{33}-\sigma_{11}\right)^{2}+H\left(\sigma_{11}-\sigma_{22}\right)^{2} \\
& +2 L \sigma_{23}^{2}+2 M \sigma_{31}^{2}+2 N \sigma_{12}^{2}=Q^{2},
\end{aligned}
$$

where $\phi\left(\sigma_{i j}\right)$ represents the current yield surface in the stress space, and $F, G, H, L, M$, and $N$ are the dimensionless constants that describe the state of plastic orthotropy. An effective yield strength parameter $Q$ can be defined to represent the characteristic size of the elastic domain at the current stress state. For isotropic materials, we can set $F=G=H=$ $L / 3=M / 3=N / 3=1 / 6$ and (2.1) reduces to the familiar Von Mises yield condition.

Let $X_{0}, Y_{0}$, and $Z_{0}$ be the initial yield stresses along the orthotropic symmetry axes and $R_{0}$, $S_{0}$, and $T_{0}$ be the initial shear yield stresses with respect to the orthotropic symmetry axes. As in Pan and Shih [11], we identify $Q$ with the shear yield stress $T$ with respect to the $x_{1}$ and $x_{2}$ axes. With this normalization, these relations follow (Hill [1]):

$$
\begin{aligned}
& G+H=\left(T_{0} / X_{0}\right)^{2}, \quad H+F=\left(T_{0} / Y_{0}\right)^{2}, \quad F+G=\left(T_{0} / Z_{0}\right)^{2} \\
& 2 L=\left(T_{0} / R_{0}\right)^{2}, \quad 2 M=\left(T_{0} / S_{0}\right)^{2}, \quad 2 N=1 .
\end{aligned}
$$

We introduce the generalized shear effective stress $\tau_{e}$ and the generalize tensile effective stress $\sigma_{e}$ by analogy with those of isotropic plasticity theories:

$$
\begin{aligned}
\tau_{e}^{2}= & \sigma_{e}^{2} / 3=F\left(\sigma_{22}-\sigma_{33}\right)^{2}+G\left(\sigma_{33}-\sigma_{22}\right)^{2}+H\left(\sigma_{11}-\sigma_{22}\right)^{2} \\
& +2 L \sigma_{23}^{2}+2 M \sigma_{31}^{2}+2 N \sigma_{12}^{2}=Q^{2} .
\end{aligned}
$$

The generalized deviatoric stress $s_{i j}$ in the direction of the normal of the yield surface is defined as

$$
s_{i j}=\partial \phi / \partial \sigma_{i j}=\partial \tau_{e}^{2} / \partial \sigma_{i j}
$$

where $s_{i j}$ reduces to the deviatoric stress for isotropic materials. 
We adopt a deformation plasticity power-law hardening relation between stresses and plastic strains as in Pan and Shih [11]:

$$
\varepsilon_{i j}^{p} / \gamma_{0}=(\alpha / 2)\left(\tau_{e} / \tau_{0}\right)^{n-1}\left(s_{i j} / \tau_{0}\right)
$$

where $n$ is the strain hardening exponent; $\tau_{0}$ is the reference shear stress, which can be identified as the initial shear yield stress of the shear stress-strain curve with respect to the $x_{1}$ and $x_{2}$ axes; $y_{0}=\tau_{0} / G$ is the associated reference shear strain, with $G$ being a representative elastic shear modulus; and $\alpha$ is the material constant particular to the present stressstrain relation. Equation (2.5) reduces to the usual $J_{2}$ power-law deformation plasticity relation for isotropic materials.

\section{Dominant singularity analysis in plane stress}

We consider a two-dimensional crack problem as depicted in Fig. 1. The Cartesian coordinates $x_{1}$ and $x_{2}$ are centered at the crack tip and the $x_{3}$ is perpendicular to the $x_{1}-x_{2}$ plane. We will confine our discussion to the cases where the Cartesian coordinate axes coincide with the symmetry axes of plastic orthotropy. The polar coordinates $\mathrm{r}$ and $\theta$ are centered at the crack tip as shown in Fig. 1. By invoking the plane-stress condition $\left(\sigma_{3 i}=0, i=1,2\right.$, or 3 ) and $2 N=1$, the yield condition (2.3) becomes

$$
F \sigma_{22}^{2}+G \sigma_{11}^{2}+H\left(\sigma_{11}-\sigma_{22}\right)^{2}+\sigma_{12}^{2}=\tau_{e}^{2}
$$

The argument leading to the HRR (Hutchinson-Rice-Rosengren) singularity field has been detailed by Hutchinson [5, 6, 14] and Rice and Rosengren [7]. Note that the elastic strain is negligible compared to the plastic strain in the immediate vicinity of the crack tip. As the orthotropic solid obeys the power-law deformation plasticity constitutive law of (2.5), the dominant asymptotic singular stresses, strains, and displacements at the crack tip can be expressed as

$$
\begin{aligned}
\sigma_{i j} & =\sigma_{0}\left(J / \alpha \sigma_{0} \varepsilon_{0} I \mathrm{r}\right)^{1 /(n+1)} \tilde{\sigma}_{i j}(\theta ; n, F, G, H ; m) \\
\varepsilon_{i j}^{p} & =\alpha \varepsilon_{0}\left(J / \alpha \sigma_{0} \varepsilon_{0} I \mathrm{r}\right)^{n /(n+1)} \tilde{\varepsilon}_{i j}(\theta ; n, F, G, H ; m) \\
u_{i}-\hat{u}_{i} & =\alpha \varepsilon_{0} r\left(J / \alpha \sigma_{0} \varepsilon_{0} I \mathrm{r}\right)^{n /(n+1)} \tilde{u}_{i}(\theta ; n, F, G, H ; m) .
\end{aligned}
$$

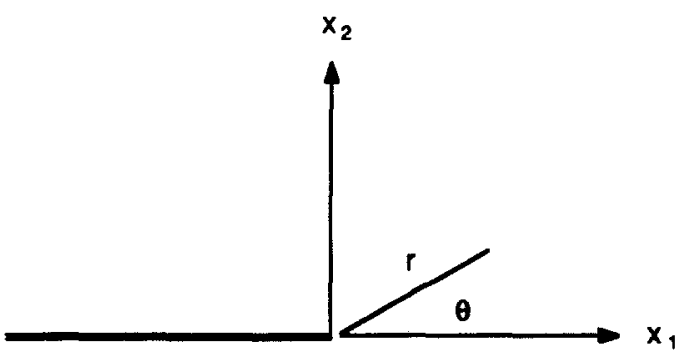

Fig. 1. Conventions at the crack tip. 
In (3.2), $\sigma_{0}$ and $\varepsilon_{0}$ are related to $\tau_{0}$ and $\gamma_{0}$ in (2.5) by $\sigma_{0}=\sqrt{3} \tau_{0}$ and $\varepsilon_{0}=\gamma_{0} / \sqrt{3}$. The normalized dimensionless functions $\tilde{\sigma}_{i j}, \tilde{\sigma}_{e}, \tilde{\varepsilon}_{i j}$, and $\tilde{u}_{i}$ depend upon the angle $\theta$, the strain hardening exponent $n$, the orthotropic material constants: $F, G$, and $H$, and the mode parameter $m$ (mode I, mode II, or mixed-mode). The dimensionless constant $I$ depends upon the material parameters $n, F, G, H$, and the mode parameter $m$. The constant $\hat{u}_{i}$ allows for a possible rigid body motion of the crack tip itself. The angular functions are normalized by setting the maximum value of $\tilde{\sigma}_{e}$ to unity. With this normalization, the present angular functions for $F=G=H=1 / 6$ are exactly the functions given by Hutchinson $[5,6]$ and Shih $[8,16]$. The $\theta$-variation of the normalized effective stress, $\tilde{\tau}_{e}$ and $\tilde{\sigma}_{e}$, are defined as

$$
\begin{aligned}
\tilde{\tau}_{e}^{2}= & \tilde{\sigma}_{e}^{2} / 3=F\left(\tilde{\sigma}_{\mathrm{rr}} \sin ^{2} \theta+\tilde{\sigma}_{\theta \theta} \cos ^{2} \theta+\tilde{\sigma}_{r \theta} \sin 2 \theta\right)^{2} \\
& +G\left(\tilde{\sigma}_{r r} \cos ^{2} \theta+\tilde{\sigma}_{\theta \theta} \sin ^{2} \theta-\tilde{\sigma}_{r \theta} \sin 2 \theta\right)^{2}+H\left[\left(\tilde{\sigma}_{r r}-\tilde{\sigma}_{00}\right) \cos 2 \theta\right. \\
& \left.-2 \tilde{\sigma}_{r \theta} \sin 2 \theta\right]^{2}+(1 / 4)\left[\left(\tilde{\sigma}_{r r}-\tilde{\sigma}_{0 \theta}\right) \sin 2 \theta+2 \tilde{\sigma}_{r \theta} \cos 2 \theta\right]^{2}
\end{aligned}
$$

The normalized strain $\tilde{\varepsilon}_{i j}$ is related to the normalized deviatoric stress $\tilde{s}_{i j}$ as

$$
\tilde{\varepsilon}_{i j}=(3 / 2) \tilde{\sigma}_{e}^{n-1} \tilde{s}_{i j}
$$

and the normalized deviatoric stress $\tilde{s}_{i j}$ is

$$
\tilde{s}_{i j}=\partial \tilde{\tau}_{e}^{2} / \partial \tilde{\sigma}_{i j}
$$

The dimensionless constant $I$ is defined by

$$
\begin{aligned}
I= & \int_{-\pi}^{\pi}\left\{(n /(n+1)) \tilde{\sigma}_{e}^{n+1} \cos \theta-\left[\operatorname { s i n } \theta \left(\tilde{\sigma}_{r r}\left(\tilde{u}_{\theta}-\tilde{u}_{r}\right)-\tilde{\sigma}_{r \theta}\left(\tilde{u}_{r}+\tilde{u}_{\theta}\right)\right.\right.\right. \\
& \left.\left.+(1 /(n+1)) \cos \theta\left(\tilde{\sigma}_{r r} \tilde{u}_{r}+\tilde{\sigma}_{r \theta} \tilde{u}_{\theta}\right)\right]\right\} \mathrm{d} \theta,
\end{aligned}
$$

where the superscript ( $)^{\circ}$ denotes the differentiation with respect to $\theta$.

The numerical procedure to determine the dependence of the singularity field on $\theta$ for a given material is outlined here. A fourth-order ordinary differential equation in terms of a separable Airy stress function can be obtained from the compatibility equation and the constitutive equation. Then, the ordinary differential equation is integrated by the fourthfifth order Runge-Kutta scheme with error and step-size control. The shooting method is employed to satisfy the stress-free boundary conditions at $\theta=180^{\circ}$ (or $-180^{\circ}$ ) and the symmetry condition at $\theta=0^{\circ}$ for pure mode I crack-tip fields.

\section{Mode I crack-tip fields}

Plane-stress crack-tip fields, as expressed in (3.2), are functions of three plastic orthotropy constants: $F, G$, and $H$. The yield condition in plane-stress has more plastic orthotropy parameters than that in plane-strain. In the latter case, the angular functions of the crack-tip fields are members of a single family parameterized by a plastic orthotropy parameter $p$ [11]. 
Here, only four simple cases are examined in detail and the presentation of the results requires a large number of graphs.

Since we assume that the plastic orthotropy remains unchanged under deformation, the crack-tip fields therefore can be expressed as functions of the relative magnitudes of the initial yield stresses $X_{0}, Y_{0}, Z_{0}$, and $T_{0}$ (see (2.2)). In this study we vary the relative magnitudes of the initial yield stresses to investigate the effects of plastic orthotropy and plastic hardening on the crack-tip fields. We only examine four simple cases where the initial yield strength in one orthotropic symmetry direction is relatively stronger or weaker than those of the others. Due to the symmetry of the mode I crack-tip fields, we concentrate our discussions to the crack-tip fields between $\theta=0^{\circ}$ and $\theta=180^{\circ}$.

\subsection{Case 1}

We first examine the effects of plastic orthotropy on the crack-tip fields when the strength in the $x_{1}$ direction is increased while the strength in the other directions remains unchanged, as for isotropic materials, i.e., $X_{0} / Y_{0}>1$ and $Y_{0}=Z_{0}=\sqrt{3} T_{0}$. Figure 2 shows the asymptotic crack-tip stress and strain fields for $n=3$, which represents materials with a relatively high hardening capacity. In Fig. 2(a), the crack-tip stress and strain fields for isotropic materials $\left(X_{0}=Y_{0}=Z_{0}=\sqrt{3} T_{0}\right)$ are shown. These fields are the same fields presented by Hutchinson [6] and Shih [8, 16]. Figures 2(b) and 2(c) show the crack-tip stress and strain fields for $X_{0} / Y_{0}=2$ and 4 , respectively.

As shown in these figures, when the strength in the $x_{1}$ direction is increased, the angular variation of $\tilde{\sigma}_{r r}$ and $\tilde{\varepsilon}_{00}$ peaks at $\theta=0^{\circ}$; a rapid change of $\tilde{\sigma}_{r r}$ occurs where the angle $\theta$ increases from $150^{\circ}$ to $170^{\circ}$. At this location of rapid change of the radial stress, the effective stress $\tilde{\sigma}_{e}$ decreases.

Figure 3 shows the asymptotic crack-tip stress and strain fields for $n=20$, which represents materials with a relatively low hardening capacity. In Fig. 3(a), the crack-tip stress and strain fields for isotropic materials are shown. Again, these fields are the same fields presented in Hutchinson [6] and Shih [8, 16]. Figures 3(b) and 3(c) show the crack-tip stress and strain fields for $X_{0} / Y_{0}=2$ and 4 , respectively. The trends of the crack-tip fields in Fig. 3 are similar to those for $n=3$ in Fig. 2. A comparison of Fig. 3 with Fig. 2 shows that as the hardening exponent $n$ increases, the radial strain $\tilde{\varepsilon}_{r r}$ approaches zero for all angles. The reason is that the limit of low-hardening solutions should approach the rigid perfectly plastic solutions, which require $\varepsilon_{r r}$ to vanish as $r$ approaches zero due to the equilibrium and the normality of plastic flow that we have assumed [17, 18]. Also, in Fig. 3, an almost discontinuous change of $\tilde{\sigma}_{r r}$ from a positive value to a negative value is shown at the location near the stress-free crack face.

The effective stress contours are plotted for $n=3$ and $n=20$ in Figs. 4(a) and 4(b), respectively, using the dimensionless similarity coordinates $\left(\alpha \tau_{0} \gamma_{0} / s J\right) x_{1}$ and $\left(\alpha \tau_{0} \gamma_{0} / s J\right) x_{2}$, where $s=\left(\tau_{0} / \tau_{\mathrm{e}}\right)^{n+1}$. As can be seen in the figures, when the strength in the $x_{1}$ direction is increased, the effective contour along the direction of $\theta=0^{\circ}$ protrudes. If the singularity field (3.2) represents the full solution everywhere within the plastic zone, then the contours in Fig. 4 represent the actual shapes of the elastic-plastic boundary. Since (3.2) usually dominates a region well within the plastic zone, these contours only suggest possible trends of the shape and the size of the plastic zone as the degree of plastic orthotropy and plastic hardening is varied. As shown in the figures, as $n$ increases, the plastic shearing becomes more 


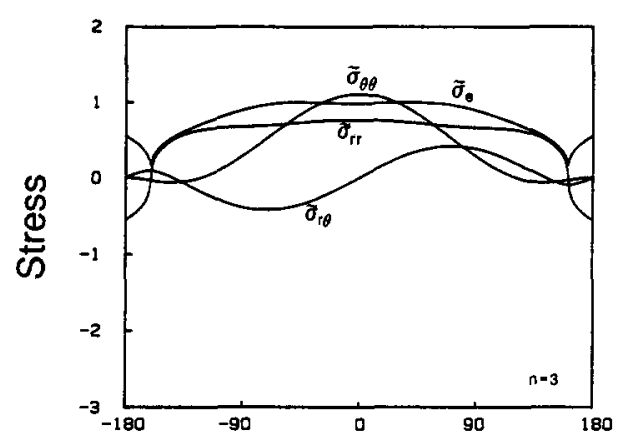

(a)
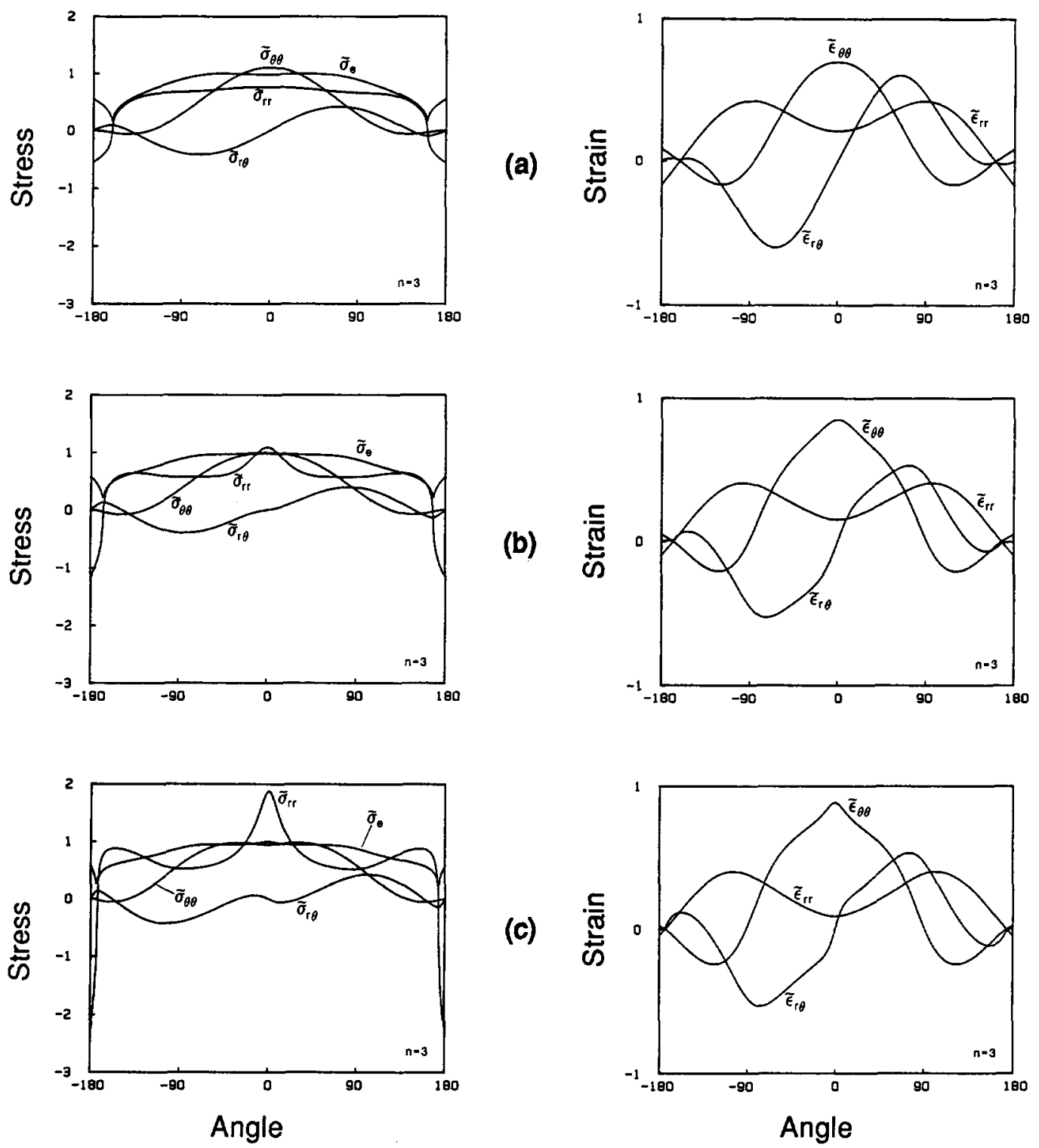

(b)

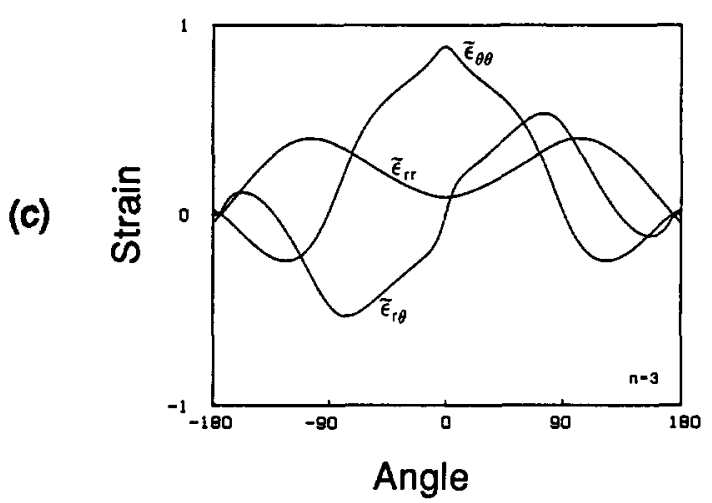

Fig. 2. Case 1: Angular distribution of the crack-tip stress and strain fields for $n=3, Y_{0}=Z_{0}=\sqrt{3} T_{0}$ and $X_{0} / Y_{0}=$ (a) 1, (b) 2 , (c) 4 .

active in front of the crack tip. However, it is possible that the shapes and the sizes of the effective stress contours in Fig. 4 could be quite different from the actual elastic-plastic boundaries.

\subsection{Case 2}

Next, we examine the effects of plastic orthotropy on the crack-tip fields when the strength in the $x_{2}$ direction is increased with respect to those of the others, i.e., $Y_{0} / X_{0}>1$ and $X_{0}=Z_{0}=\sqrt{3} T_{0}$. This case is relevant to the photoelastic study of the fields near a crack in a graphite/epoxy composite laminate by Yeow, Morris, and Brinson [15]. 

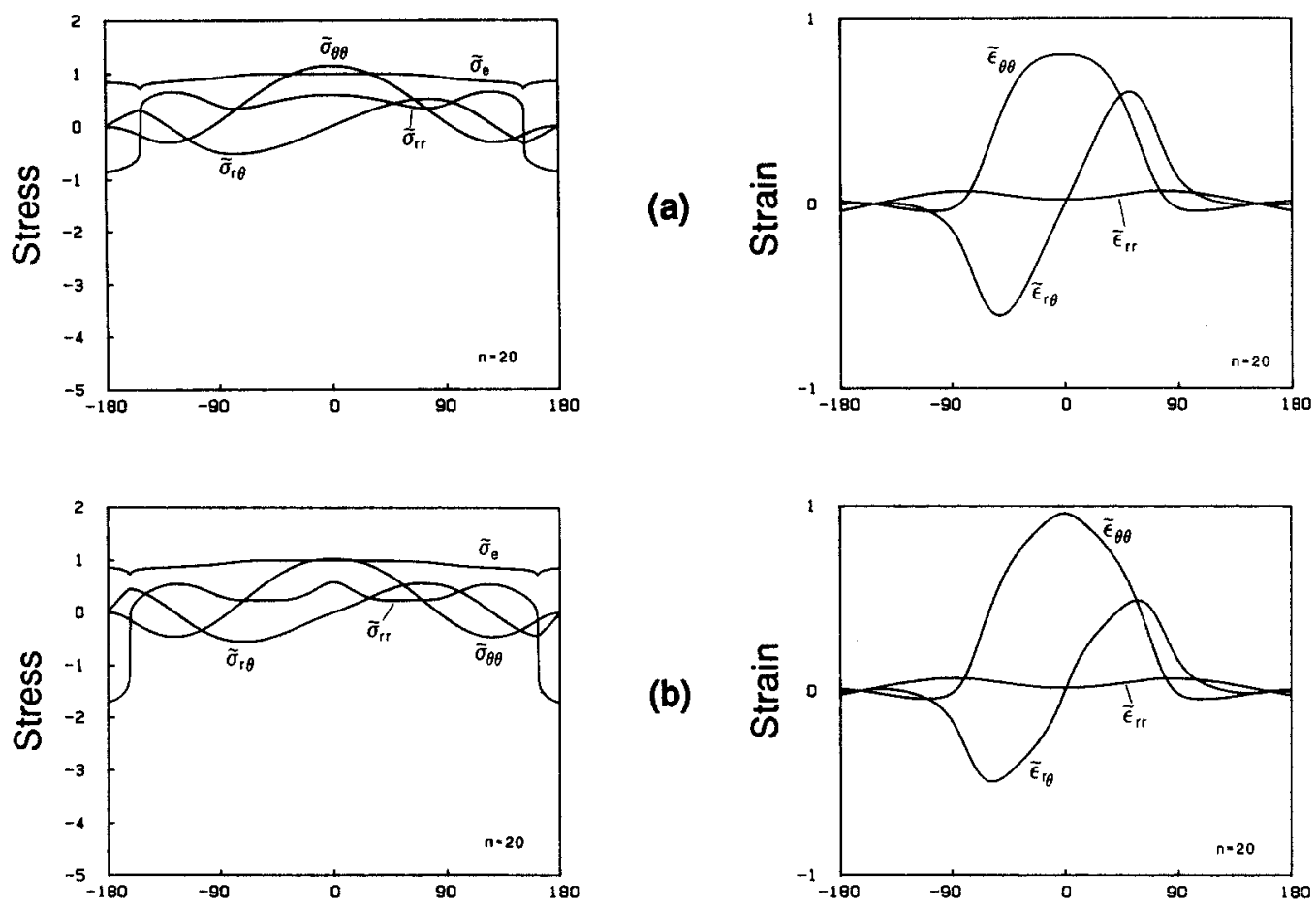

(b)
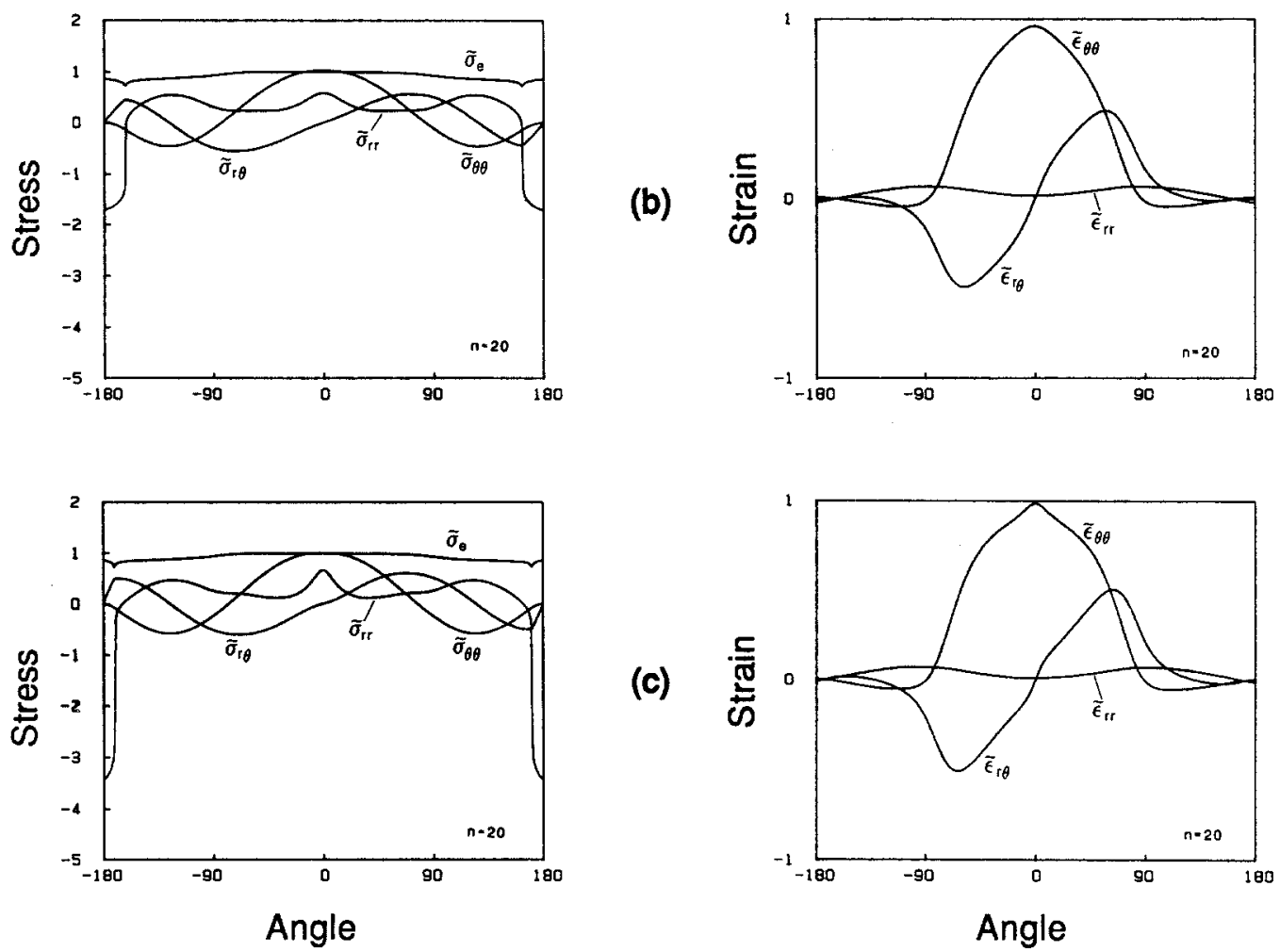

Fig. 3. Case 1: Angular distribution of the crack-tip stress and strain fields for $n=20, Y_{0}=Z_{0}=\sqrt{3} T_{0}$ and $X_{0} / Y_{0}=$ (a) 1 , (b) 2 , (c) 4 .

Figure 5 shows the asymptotic crack-tip stress and strain fields for $n=3$. Figure 5(a) represents the crack-tip stress and strain fields for isotropic materials. Figures $5(\mathrm{~b})$ and $5(\mathrm{c})$ show the crack-tip stress and strain fields for $Y_{0} / X_{0}=2$ and 4, respectively.

Figure 5 demonstrates that as the strength in the $x_{2}$ direction is increased, the angular functions $\tilde{\sigma}_{e}, \tilde{\sigma}_{r r}$, and $\tilde{\varepsilon}_{r \theta}$ peak around $\theta=90^{\circ} ; \tilde{\sigma}_{\theta \theta}$ increases substantially at $\theta=0^{\circ}$. There is a region where a rapid change of $\tilde{\sigma}_{r r}$ occurs; at this location, the effective stress decreases.

Figure 6 shows the crack-tip stress and strain fields for $n=20$. Figure 6(a) shows the crack-tip stress and strain fields for isotropic materials. Figures 6(b) and 6(c) depict the crack-tip stress and strain fields for $Y_{0} / X_{0}=2$ and 4, respectively. The trends of the crack-tip fields are similar to those for $n=3$, plotted in Fig. 5. A comparison of Fig. 6 with Fig. 5 shows 


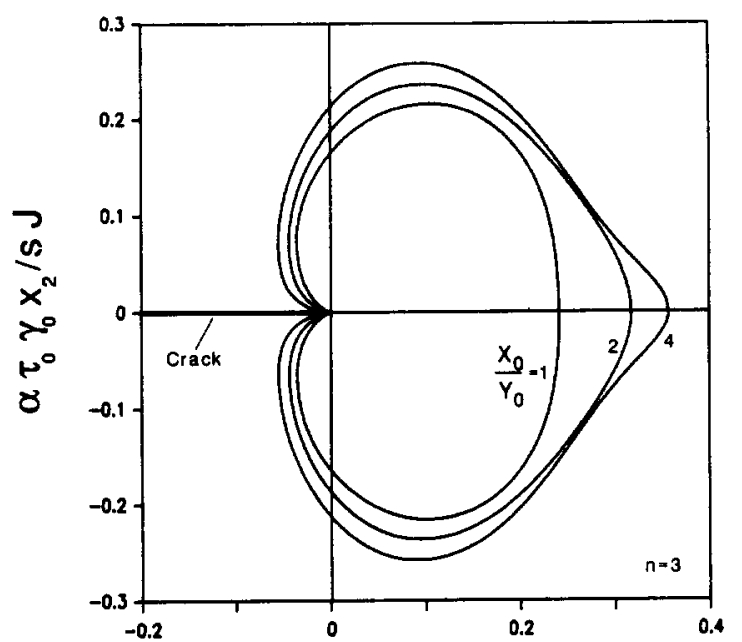

(a)

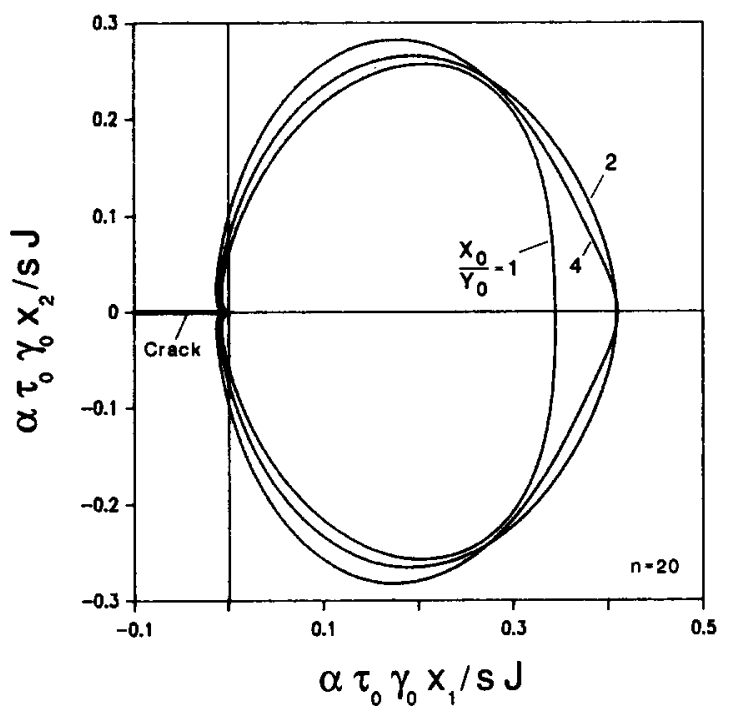

(b)

Fig. 4. Case 1: Effective stress contours plotted using the similarity coordinates for $Y_{0}=Z_{0}=\sqrt{3} T_{0}$ and $X_{0} / Y_{0}=1,2$, and 4. (a) $n=3$, (b) $n=20$.

that as the hardening exponent $n$ increases, the angular variation of $\tilde{\sigma}_{r r}$ near the stress-free crack face resembles a discontinuity, $\tilde{\varepsilon}_{r r}$ decreases to zero for all angles, and $\tilde{\varepsilon}_{r \theta}$ near $\theta=90^{\circ}$ resembles a delta function for $Y_{0} / X_{0}=4$. The angle $\theta$ where the apparent discontinuity of the radial stress occurs only increases a bit as the strength in the $x_{2}$ direction is increased.

The effective stress contours for $n=3$ and $n=20$ are plotted in Fig. 7(a) and 7(b), respectively. As these figures show, when the strength in the $x_{2}$ direction is increased, the effective stress contour becomes elongated in the $x_{2}$ direction. This is similar to the planestrain case for $p<1$, where $p$ is the plastic orthotropy parameter defined by Pan and Shih [11]. However, the nature of the plane-stress crack-tip field differs from that of the planestrain crack-tip field and this is best demonstrated by the asymptotic crack-tip solutions for 

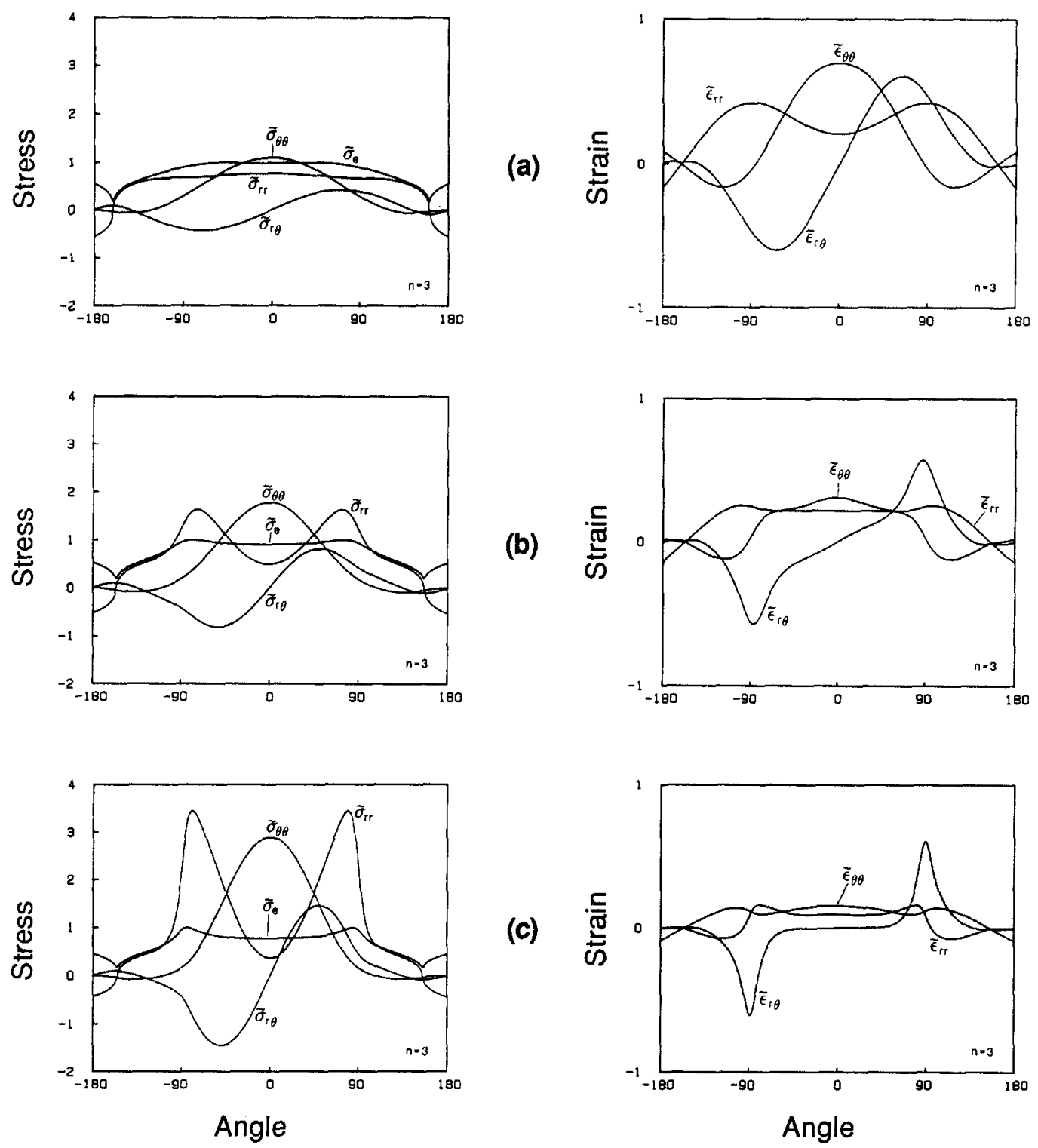

Fig. 5. Case 2: Angular distribution of the crack-tip stress and strain fields for $n=3, X_{0}=Z_{0}=\sqrt{3} T_{0}$ and $Y_{0} / X_{0}=$ (a) 1 , (b) 2 , (c) 4 .

perfectly plastic isotropic materials. According to the results of the asymptotic analysis for perfectly plastic isotropic materials within the context of the small strain approach, under the small-scale yielding condition, the plane-stress case has a fan sector directly ahead of a crack tip [6]. This fan sector may induce a large amount of plastic deformation ahead of the crack tip. On the contrary, the plane-strain case has a constant stress sector directly ahead of a crack tip. This constant stress sector only induces a limited amount of plastic shearing [19-22].

The fracture behavior of cracked graphite/epoxy laminates has been studied by Yeow, Morris, and Brinson [15]. They used photoelastic coatings to reveal the intense-stress regions near the crack tips in several graphite/epoxy laminates. For the $\left[0^{\circ}\right]_{8 s}$ laminate, the intensestress region appears as two narrow strips below and above the crack tip. The final fracture 


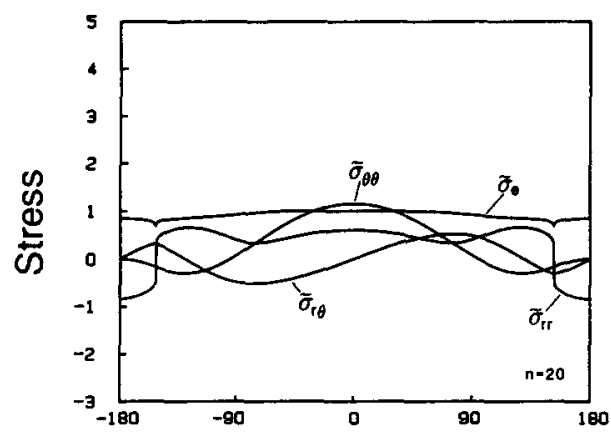

(a)
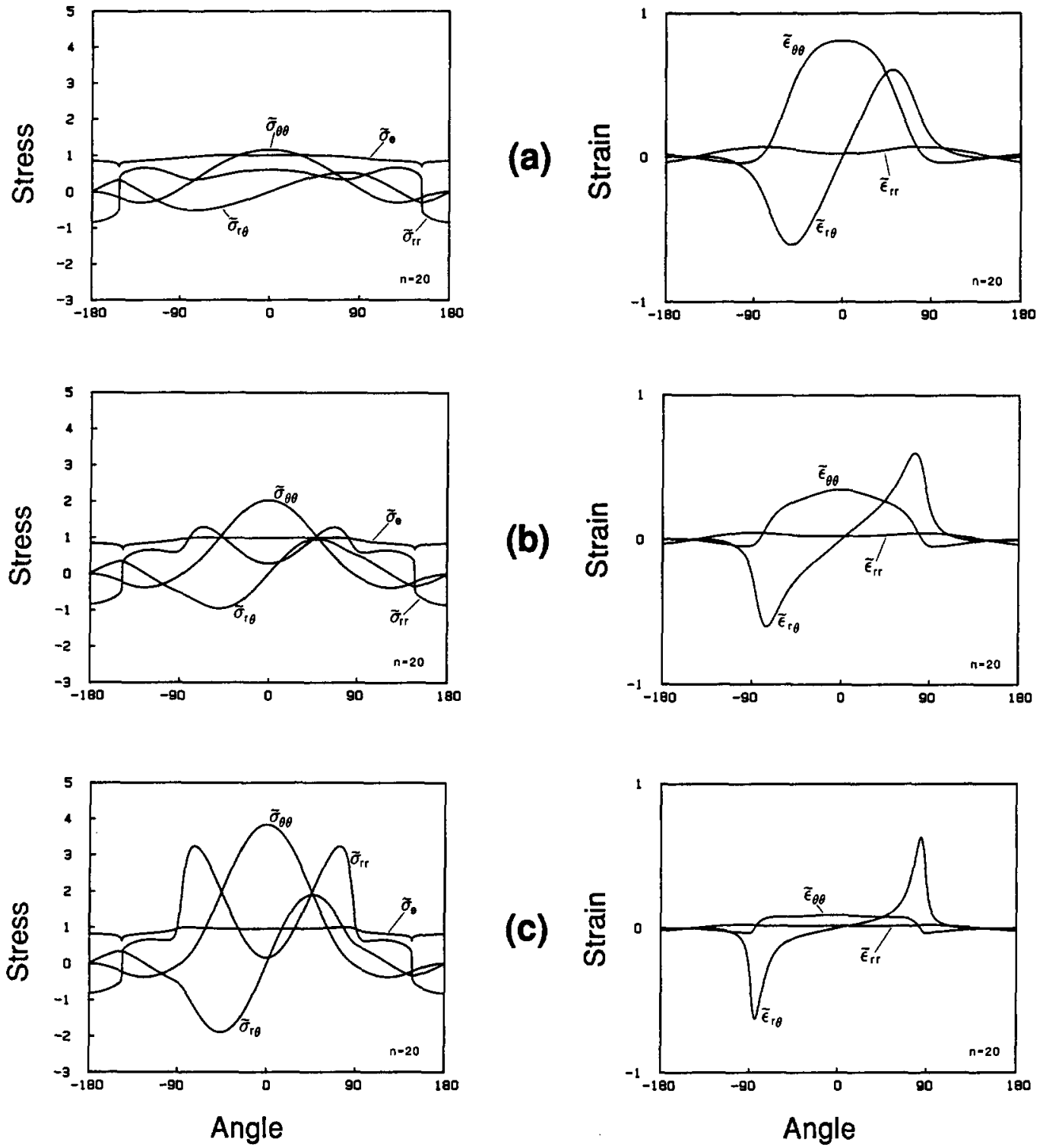

Fig. 6. Case 2: Angular distribution of the crack-tip stress and strain fields for $n=20, X_{0}=Z_{0}=\sqrt{3} T_{0}$ and $Y_{0} / X_{0}=$ (a) 1 , (b) 2 , (c) 4 .

mode for the cracked laminate is axial splitting in the $90^{\circ}$ and $-90^{\circ}$ directions (the fiber direction).

Suppose that the nonlinear behavior of the composite can be qualitatively approximated on a macroscopic scale by the power-law stress-strain relation described here. Note that in this study the effective stress contours plotted as functions of plastic orthotropy parameters indicate the trend of the intense-stress region near the crack tip as the plastic orthotropy parameters are varied. As shown in Fig. 7 for $Y_{0} / X_{0}=1,2$, and 4 with $X_{0}=Z_{0}=\sqrt{ } 3 T_{0}$, the intense deformation zone near the crack tip becomes two narrow strips below and above the crack tip as the strength in the $x_{2}$ direction is increased. 


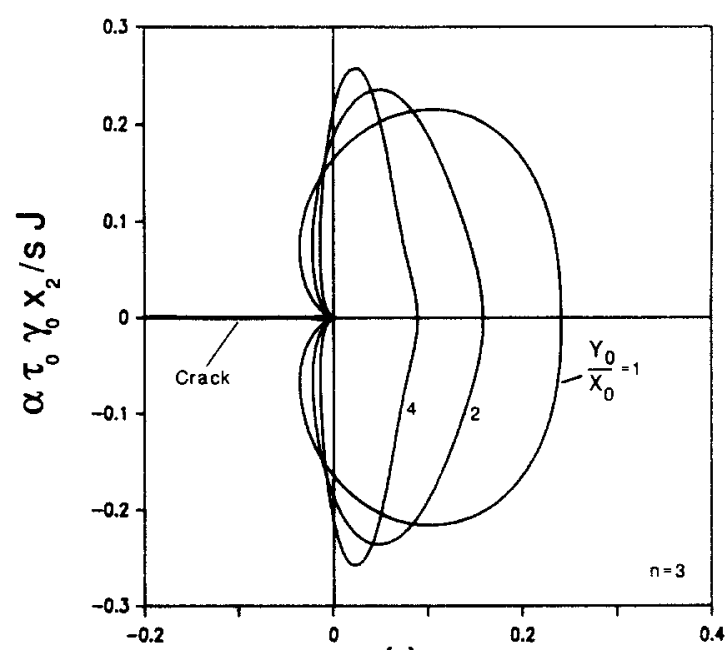

(a)

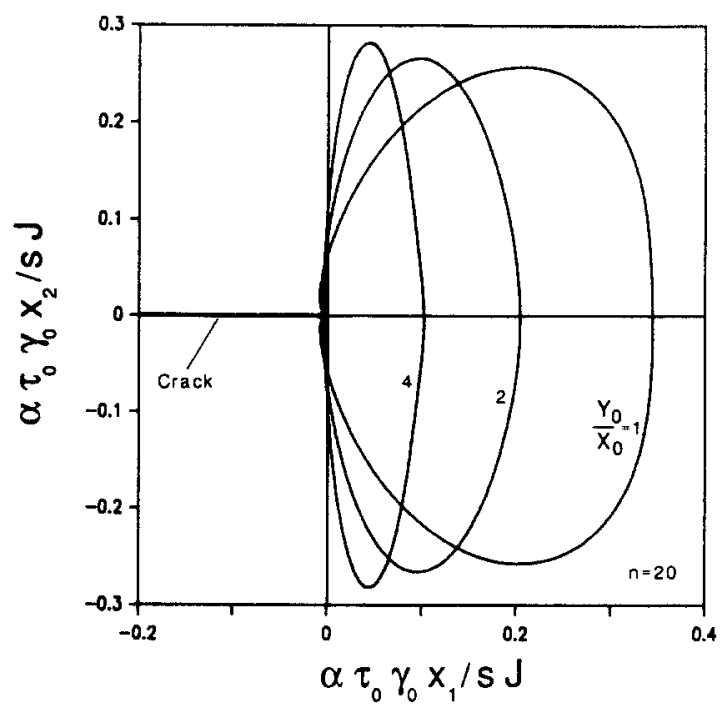

(b)

Fig. 7. Case 2: Effective stress contours plotted using the similarity coordinates for $X_{0}=Z_{0}=\sqrt{3} T_{0}$ and $Y_{0} / X_{0}=1,2$, and 4. (a) $n=3$, (b) $n=20$.

The $\left[0^{\circ}\right]_{8 s}$ laminate used in the experiment of Yeow et al. [15] exhibits a relatively larger strength in the direction perpendicular to the crack line than those in the other directions due to fiber reinforcement. As noted, the intense-stress region near the crack tip observed in their experiment appears as two narrow strips below and above the crack tip. The present continuum analysis suggests trends that are qualitatively in agreement with the observations near a macroscopic crack tip in a composite laminate. However, it is well known that the microscopic fracture processes near a macroscopic crack tip in a composite material are extremely complex and we do not suggest that the competing separation/ failure processes can be correlated to plastic zone sizes and shapes or by the HRR type fields (3.2). 


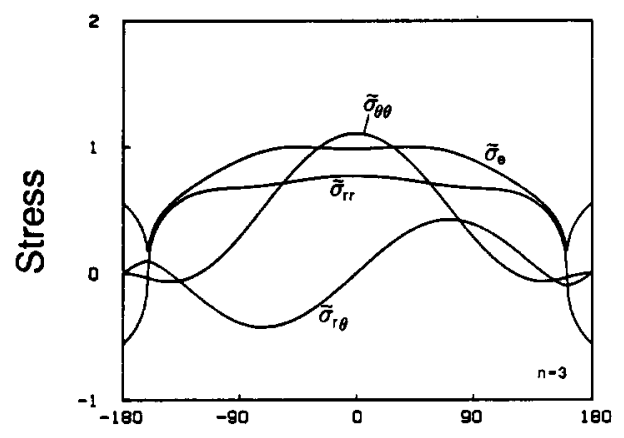

(a)
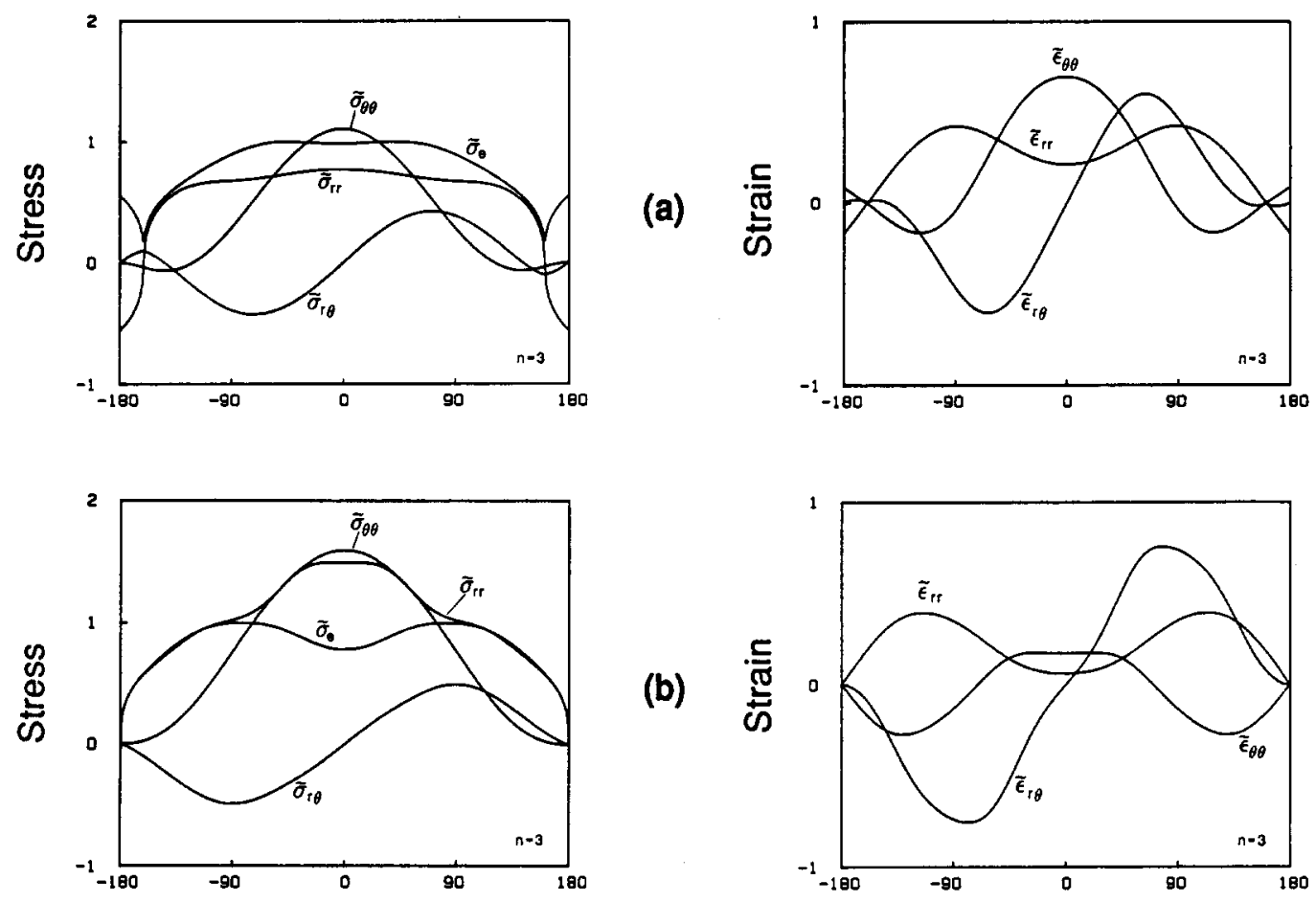

(b)
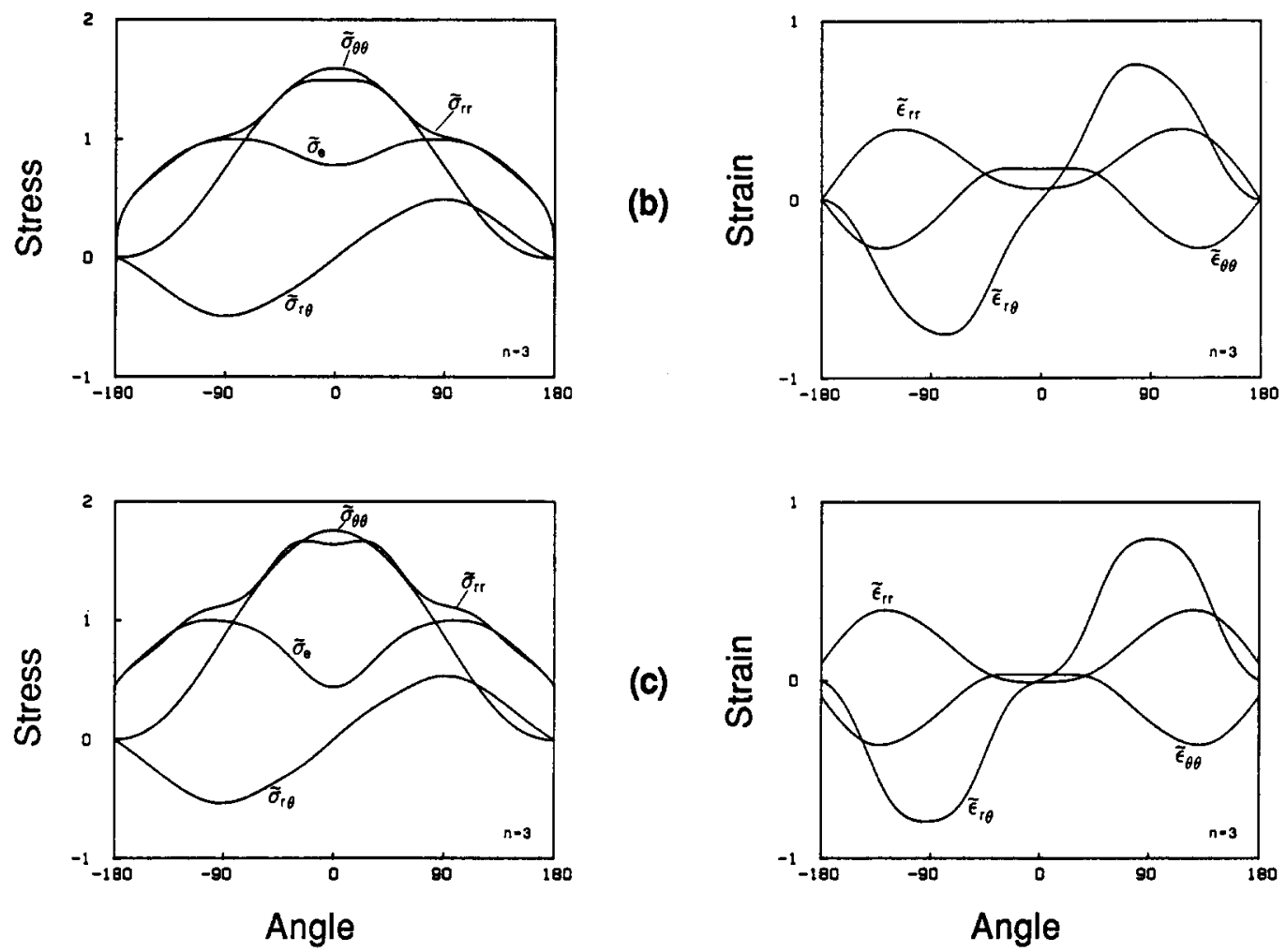

Fig. 8. Case 3: Angular distribution of the crack-tip stress and strain fields for $n=3, X_{0}=Y_{0}=\sqrt{3} T_{0}$ and $Z_{0} / X_{0}=$ (a) 1 , (b) 2 , (c) 4 .

\subsection{Case 3}

Now, we examine the effects of plastic orthotropy on the crack-tip fields when the strength in the $x_{3}$ direction is increased with respect to those of the others, i.e., $Z_{0} / X_{0}>1$ and $X_{0}=Y_{0}=\sqrt{3} T_{0}$. The transversely isotropic materials that we consider in this case are isotropic in the $x_{1}-x_{2}$ plane and have an orthotropic symmetry axis in the $x_{3}$ direction.

Figure 8 shows the asymptotic crack-tip stress and strain fields for $n=3$. Figure $8(a)$ represents the crack-tip stress and strain fields for isotropic materials. Figures 8(b) and 8(c) give the crack-tip stress and strain fields for $Z_{0} / X_{0}=2$ and 4 , respectively.

As we examine the crack-tip stress and strain fields, we find that when the strength in the $x_{3}$ direction is increased, $\sigma_{r r}$ at $\theta=180^{\circ}$ changes from a negative value to a positive value 


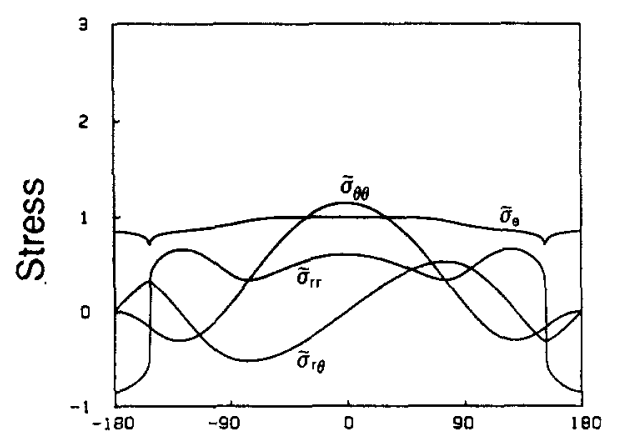

(a)
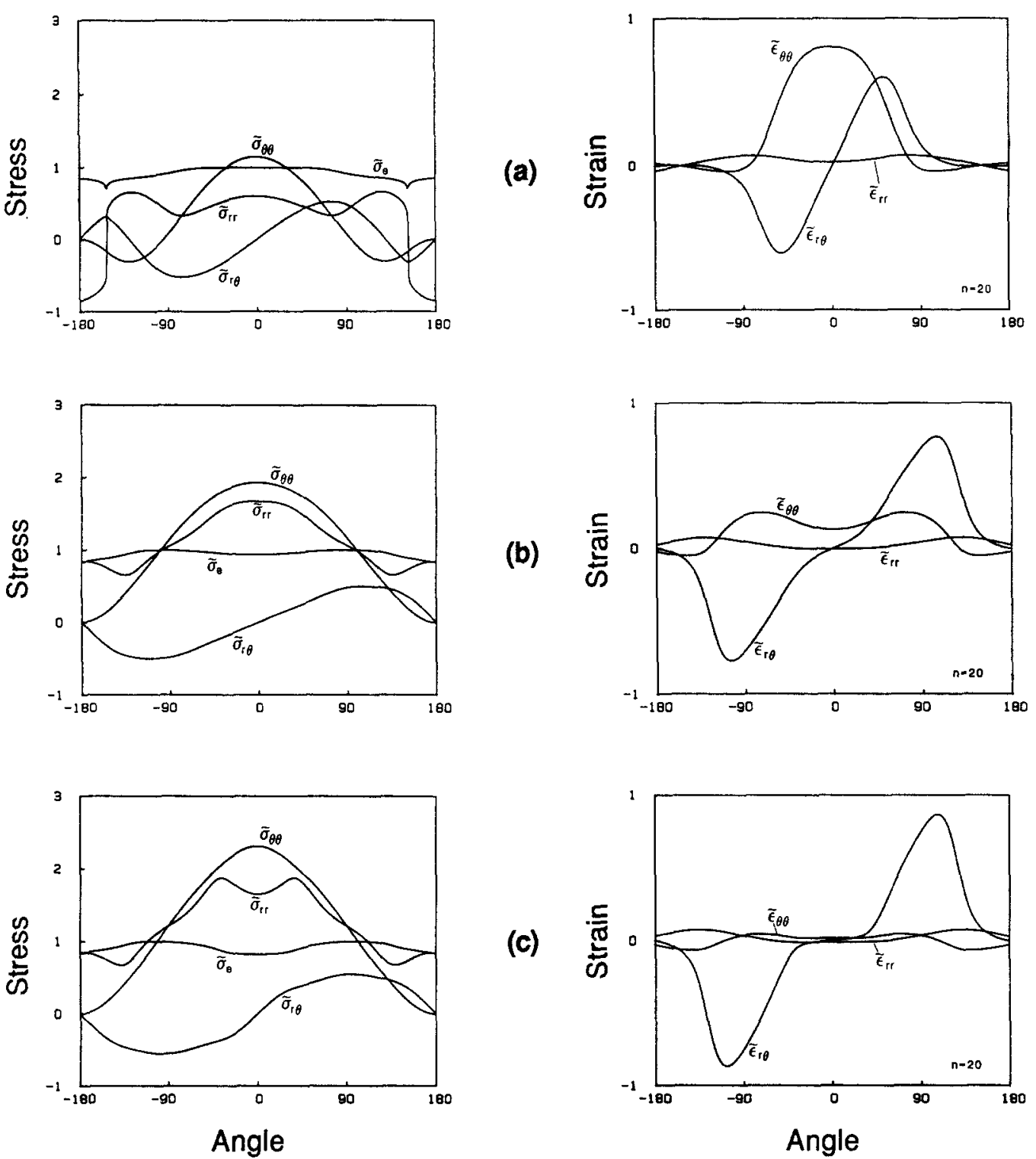

Fig. 9. Case 3: Angular distribution of the crack-tip stress and strain fields for $n=20, X_{0}=Y_{0}=\sqrt{3} T_{0}$ and $Z_{0} / X_{0}=$ (a) 1 , (b) 2 , (c) 4 .

and the rapid change of $\tilde{\sigma}_{r r}$ near the stress-free crack face disappears. As $Z_{0} / X_{0}$ becomes large, the crack-tip field tends to assume the characteristics of the plane-strain mode I crack-tip field for isotropic materials: large hydrostatic tension in front of the crack tip and large plastic shearing around $\theta=90^{\circ}$.

Here, we can argue that when we reinforce the strength in the $x_{3}$ direction, we suppress the plastic deformation in the $x_{3}$ direction. Thus, a plane-strain-like condition develops in the $x_{3}$ direction in spite of the stress-free conditions in the $x_{3}$ direction in plane-stress. Further, as the strength in the $x_{3}$ direction is increased, $\tilde{\varepsilon}_{33}$ approaches zero. The incompressibility requires that $\tilde{\varepsilon}_{r} \simeq-\tilde{\varepsilon}_{00}$ as $\tilde{\varepsilon}_{33}$ approaches zero. This trend can be seen in Fig. 8(c).

Figure 9 shows the asymptotic crack-tip stress and strain fields for $n=20$. Figure 9(a) gives the crack-tip stress and strain fields for isotropic materials. Figures $9(\mathrm{~b})$ and $9(\mathrm{c})$ show 


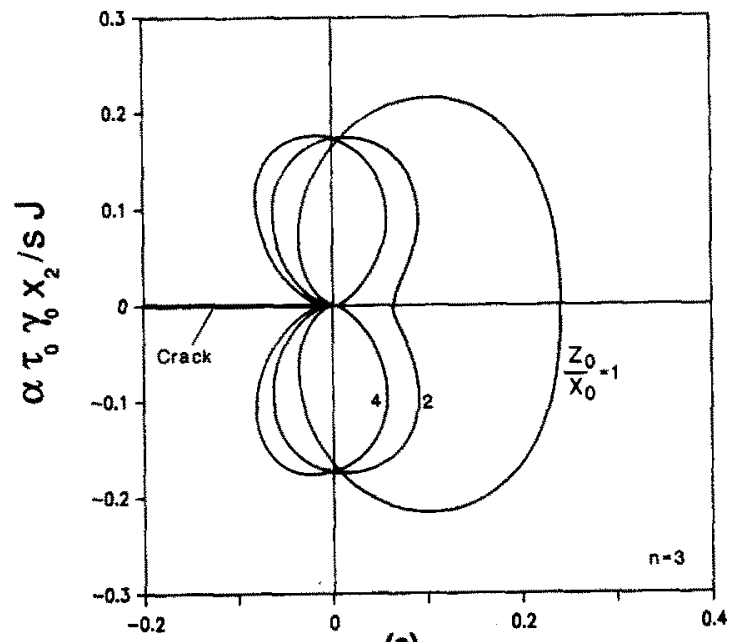

(a)

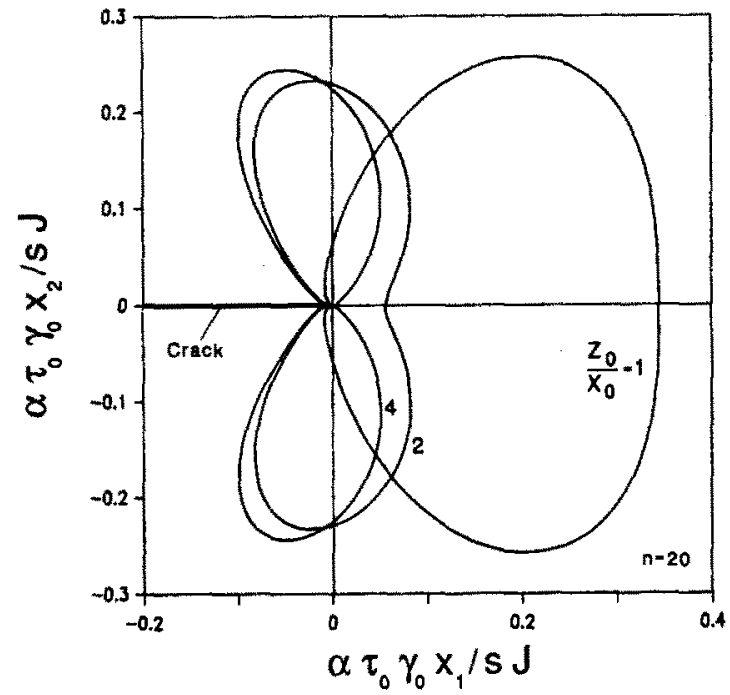

(b)

Fig. 10. Case 3: Effective stress contours plotted using the similarity coordinates for $X_{0}=Y_{0}=\sqrt{3} T_{0}$ and $Z_{0} / X_{0}=1,2$, and 4. (a) $n=3$, (b) $n=20$.

the crack-tip stress and strain fields for $Z_{0} / X_{0}=2$ and 4 , respectively. The trends of the crack-tip fields in Fig. 9 are similar to those for $n=3$, plotted in Fig. 8. These figures show that when the hardening exponent $n$ and the ratio of $X_{0} / Z_{0}$ increase, both $\tilde{\varepsilon}_{r r}$ and $\tilde{\varepsilon}_{00}$ approach zero (as demonstrated in Fig. 9(c)) due to the incompressibility, plastic orthotropy, equilibrium, and normality of plastic flow, which have been discussed earlier.

Figures 10(a) and 10(b) show the effective stress contours for $n=3$ and $n=20$, respectively. As shown in the figures, when $Z_{0} / X_{0}$ increases, the plastic deformation directly ahead of the crack tip decreases and the plastic deformation shifts to the region above and below the crack tip. In addition, the effective stress contour resembles the one for isotropic material under plane-strain conditions. 

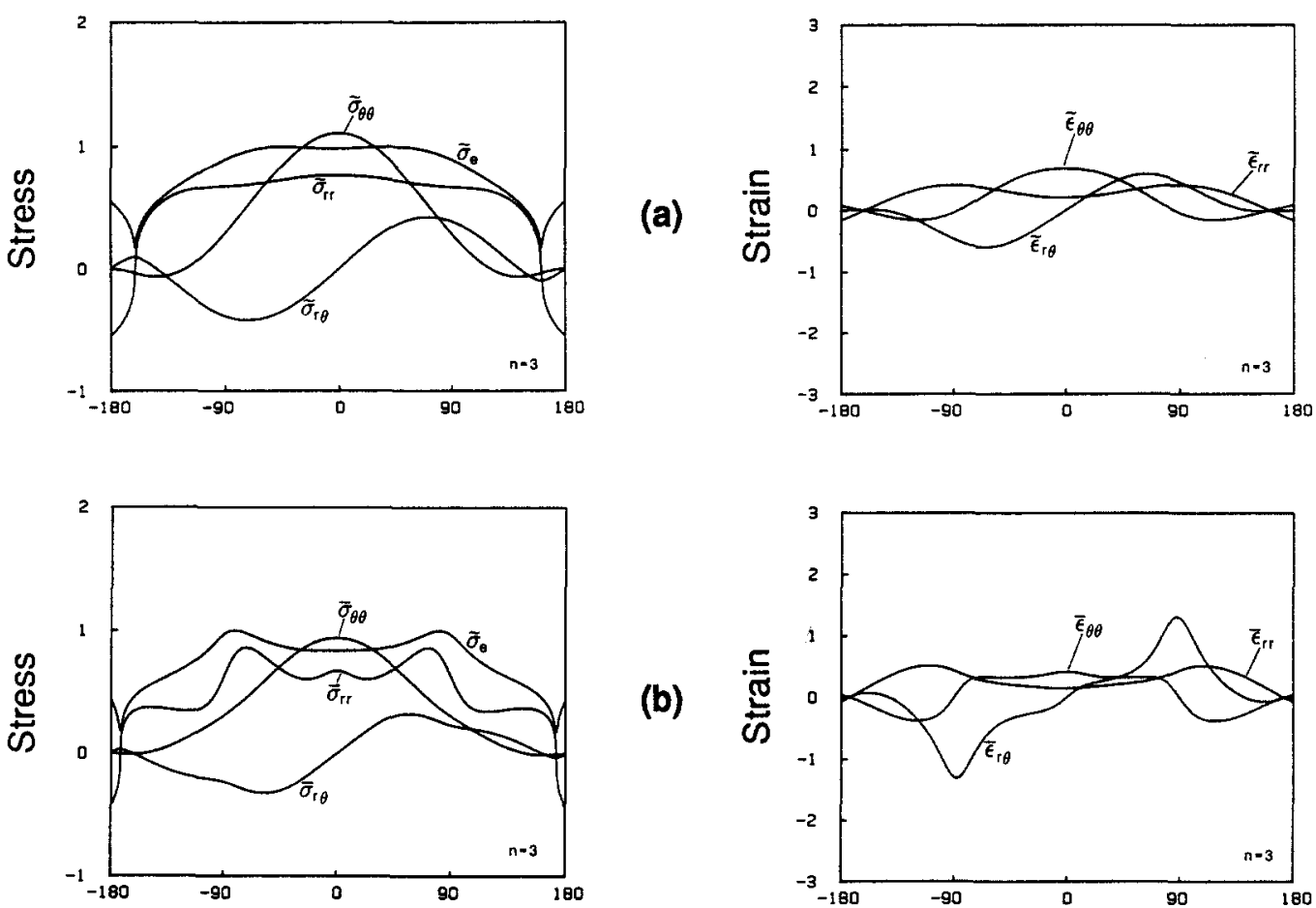

(b)
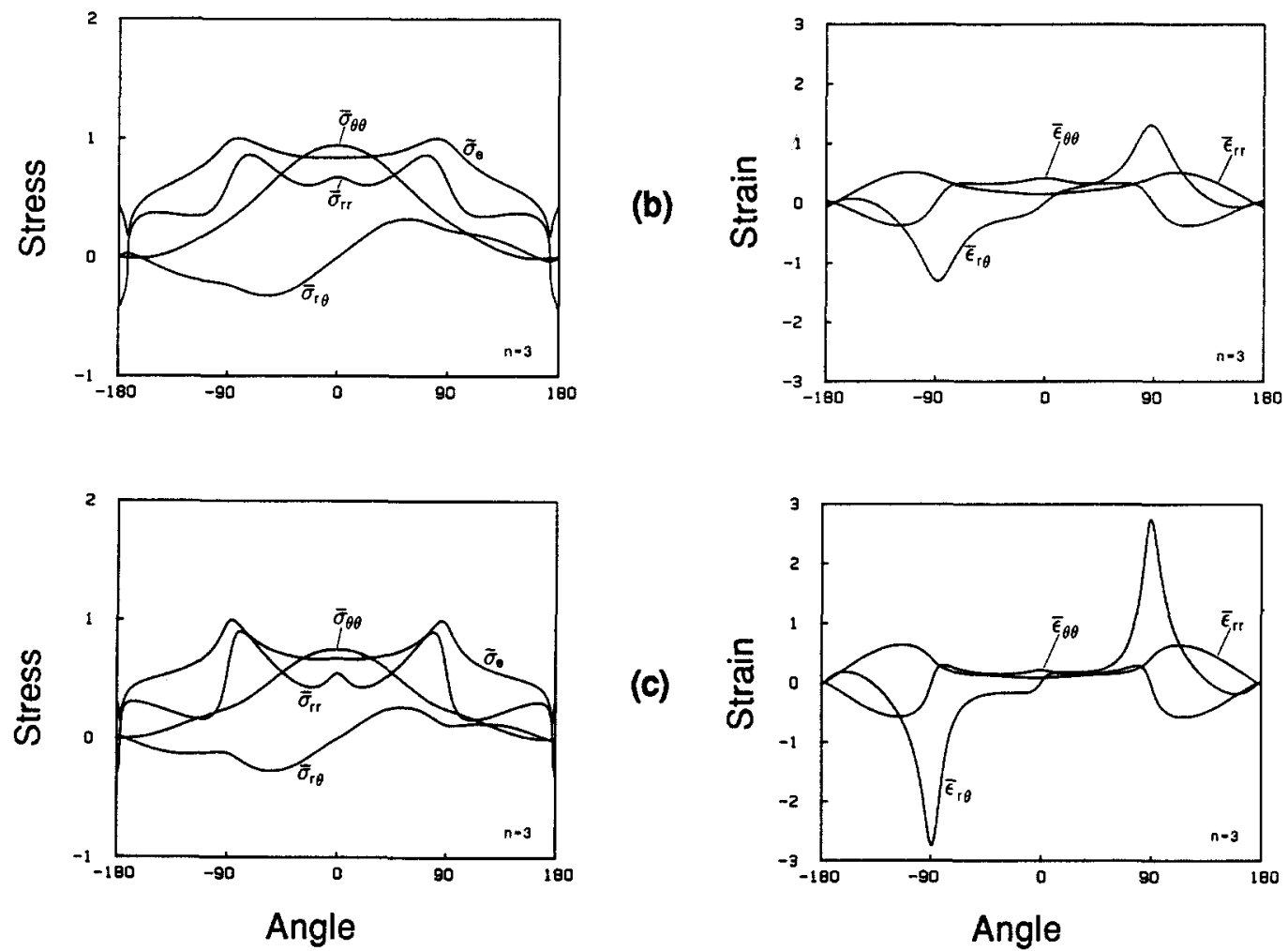

Fig. 11. Case 4: Angular distribution of the crack-tip stress and strain fields for $n=3, X_{0}=Y_{0}=Z_{0}$ and $\sqrt{3} T_{0} / X_{0}=$ (a) 1, (b) $1 / 2$, (c) $1 / 4$.

\subsection{Case 4}

Finally, we examine the effects of plastic orthotropy on the crack-tip fields when the shear strength with respect to the $x_{1}$ and $x_{2}$ axes is decreased (or increased) with respect to those of the others, i.e., $\sqrt{3} T_{0} / X_{0}<1$ (or $>1$ ) and $X_{0}=Y_{0}=Z_{0}$. Because the effective stress is defined here with respect to the in-plane shear strength, this case is equivalent to the one where the tensile strength in the $x_{1}, x_{2}, x_{3}$ directions is increased (or decreased) with respect to the in-plane shear strength.

We examine the case where the in-plane shear strength is decreased. Figure 11 shows the asymptotic crack-tip stress and strain fields for $n=3$. Figure 11(a) gives the crack-tip 


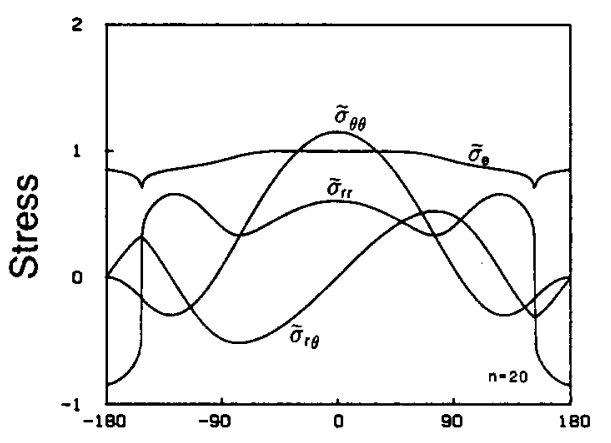

(a)
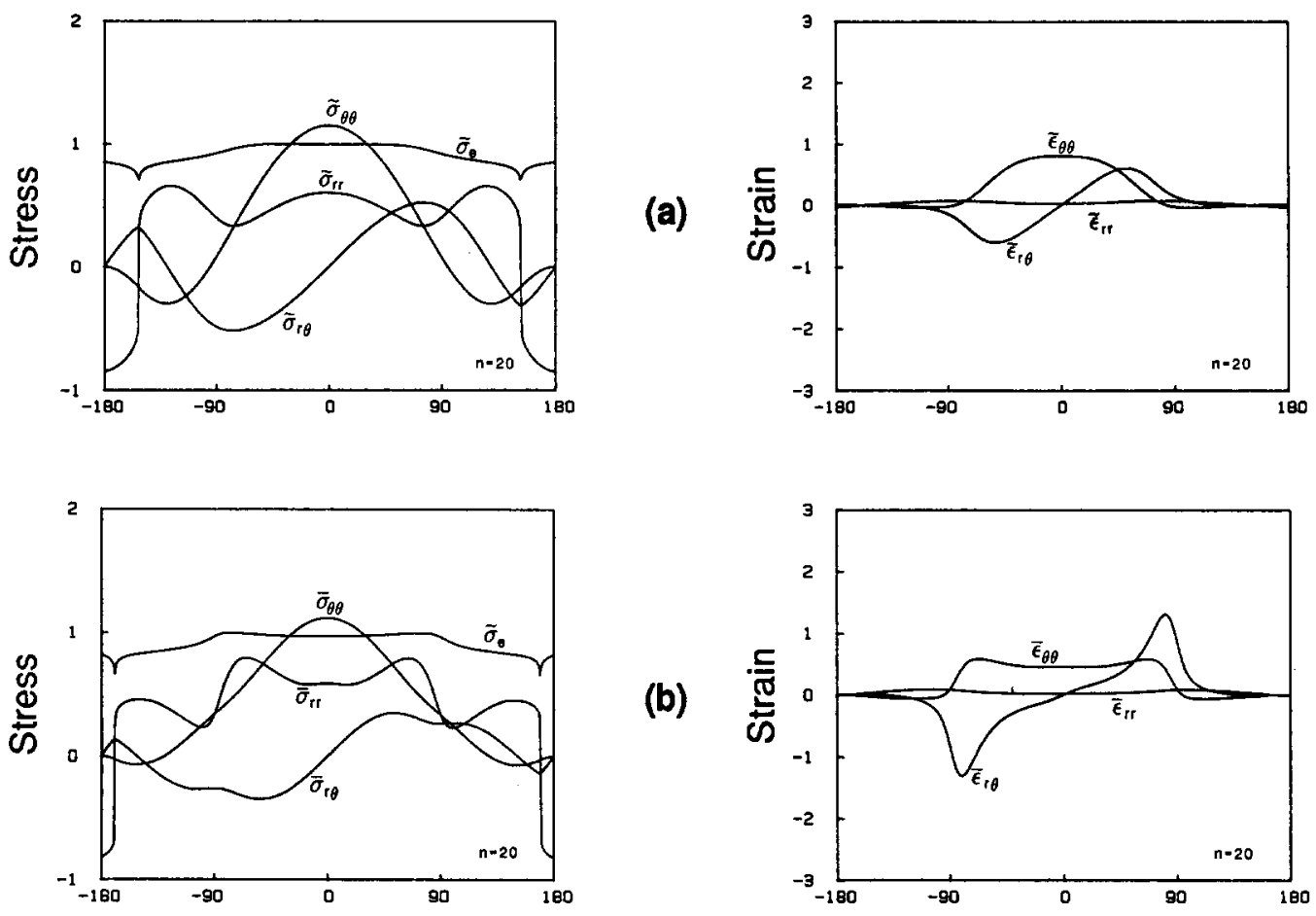

(b)
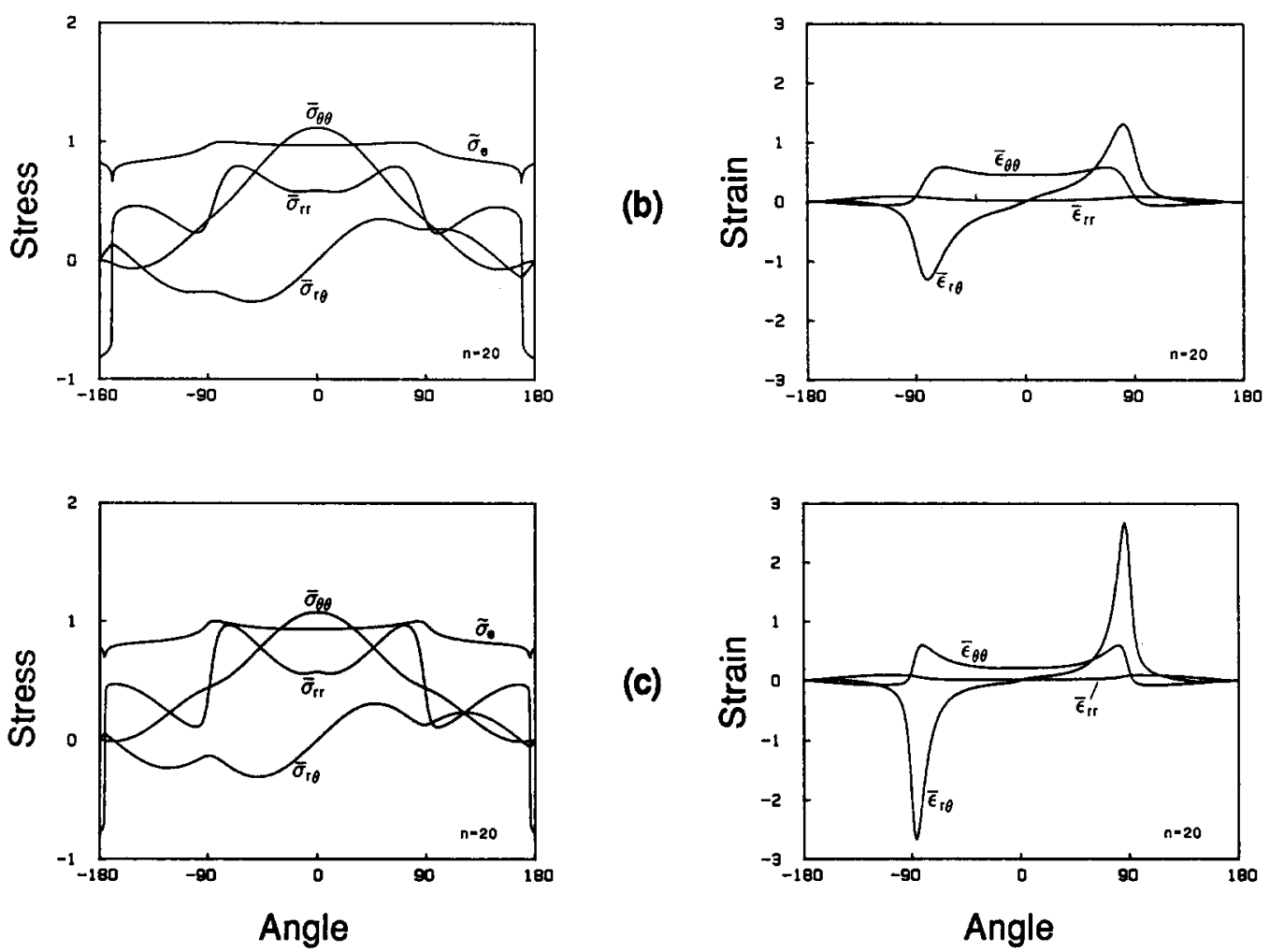

Fig. 12. Case 4: Angular distribution of the crack-tip stress and strain fields for $n=20, X_{0}=Y_{0}=Z_{0}$ and $\sqrt{3} T_{0} / X_{0}=$ (a) 1, (b) $1 / 2$, (c) $1 / 4$.

stress and strain fields for isotropic materials. Figures 11(b) and 11(c) show the cracktip stress and strain fields for $\sqrt{3} T_{0} / X_{0}=1 / 2$ and $1 / 4$, respectively. Note that the dimensionless quantities in the plots are defined by $\bar{\sigma}_{i j}=\left(\sqrt{3} T_{0} / X_{0}\right) \tilde{\sigma}_{i j}$ and $\bar{\varepsilon}_{i j}=\tilde{\varepsilon}_{i j} /\left(\sqrt{3} T_{0} / X_{0}\right)$. These figures demonstrate the trend that as the in-plane shear strength is decreased (or the tensile strength in the $x_{1}, x_{2}$, and $x_{3}$ directions is increased), the magnitude of the angular functions $\tilde{\sigma}_{r r}, \tilde{\sigma}_{r \theta}$, and $\tilde{\sigma}_{\theta \theta}$ becomes large, and the angular functions $\tilde{\sigma}_{e}$ and $\tilde{\varepsilon}_{r \theta}$ peak around $\theta=90^{\circ}$.

Figure 12 shows the asymptotic crack-tip stress and strain fields for $n=20$. Figure 12(a) gives the crack-tip stress and strain fields for isotropic materials. Figures 12 (b) and 12(c) show the crack-tip stress and strain fields, $\bar{\sigma}_{i j}$ and $\bar{\varepsilon}_{i j}$, for $\sqrt{3} T_{0} / X_{0}=1 / 2$ and $1 / 4$, respectively. 


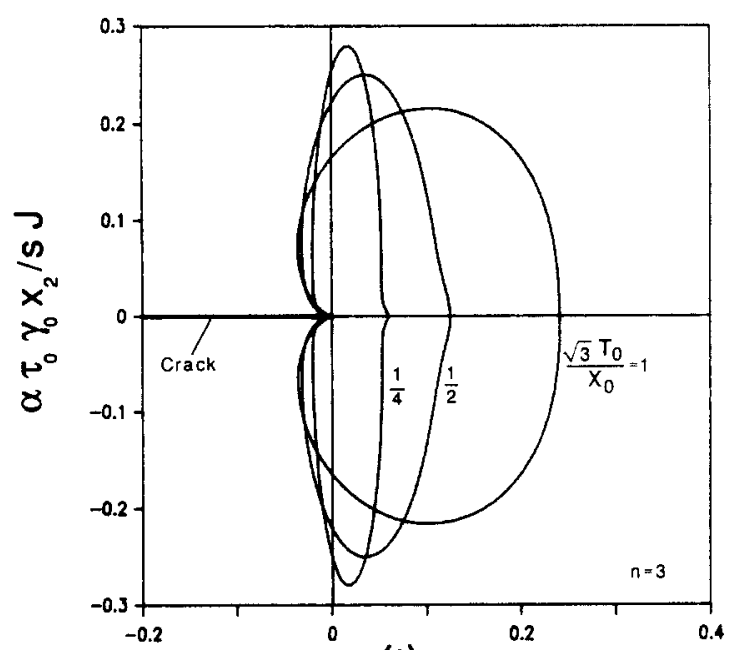

(a)

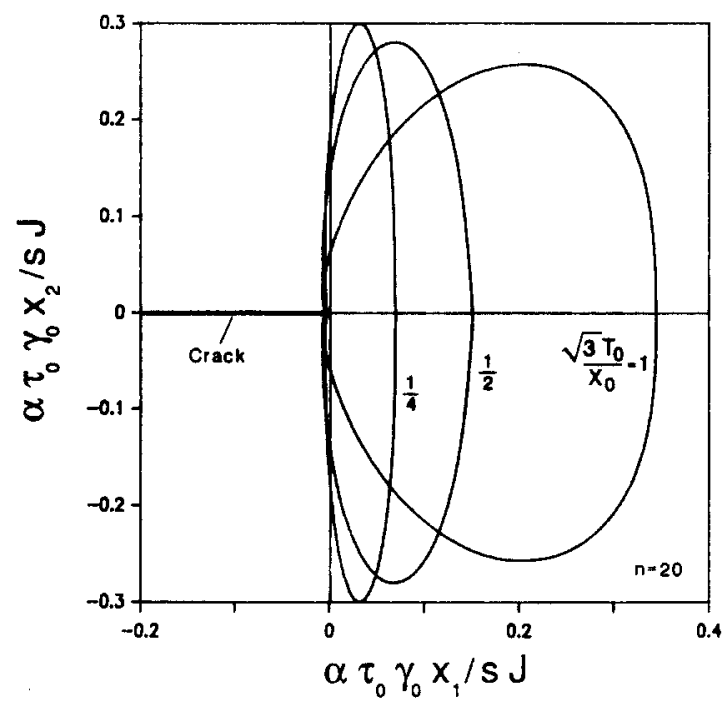

(b)

Fig. 13. Case 4: Effective stress contours plotted using the similarity coordinates for $X_{0}=Y_{0}=Z_{0}$ and $\sqrt{3} T_{0} / X_{0}=1,1 / 2$, and $1 / 4$. (a) $n=3$, (b) $n=20$.

The trends of the crack-tip fields in Fig. 12 are similar to those for $n=3$ in Fig. 11. Note that when the in-plane shear strength is decreased (or the tensile strength in the $x_{1}, x_{2}$, and $x_{3}$ directions is increased), the angular function $\tilde{\varepsilon}_{r \theta}$ at $\theta=90^{\circ}$ resembles a delta function for both $n=3$ and $n=20$, as was observed for Case 2, where only the tensile strength in the $x_{2}$ direction is increased.

Figures 13(a) and 13(b) show the effective stress contours for $n=3$ and $n=20$, respectively. The effective stress contour is elongated in the $x_{2}$ direction as the in-plane shear strength is decreased. This shape change of the effective stress contour as the in-plane shear strength is decreased (or the tensile strength in the $x_{1}, x_{2}$, and- $x_{3}$ directions is increased) is similar to that of Case 2, where only the tensile strength in the $x_{2}$ direction is increased. 

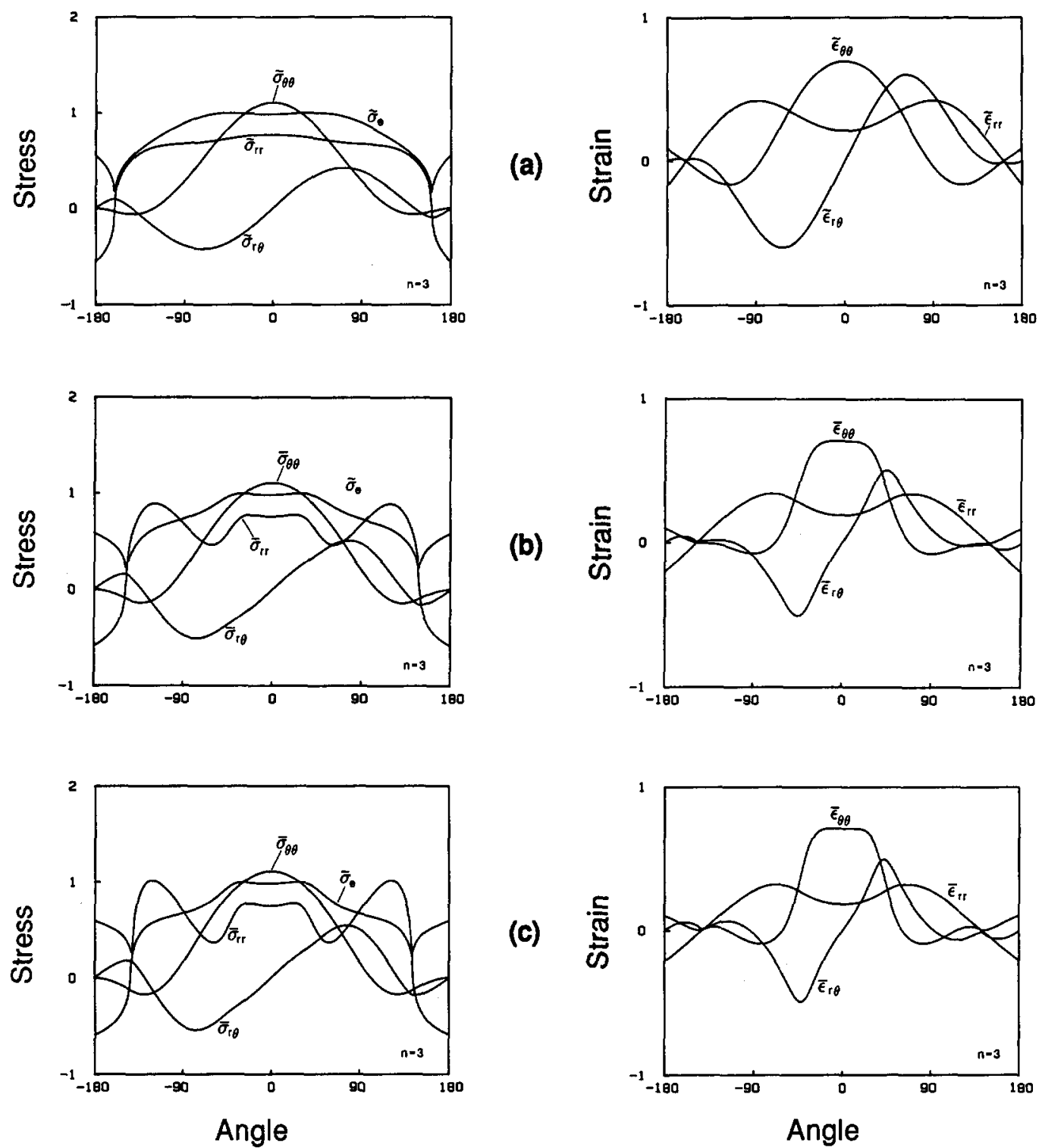

(b)

(a)

(c)

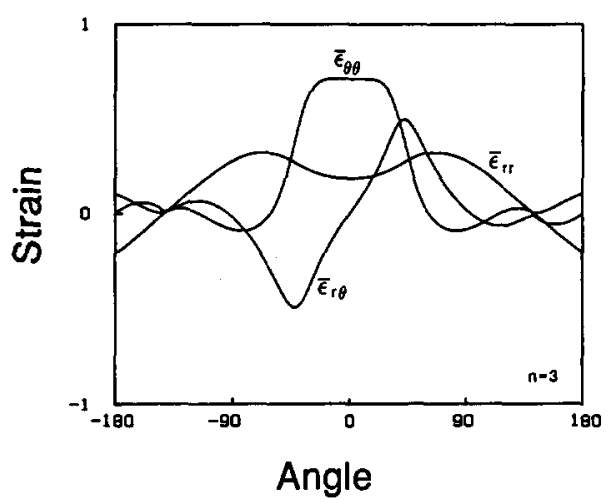

Fig. 14. Case 4: Angular distribution of the crack-tip stress and strain fields for $n=3, X_{0}=Y_{0}=Z_{0}$ and $\sqrt{3} T_{0} / X_{0}=$ (a) 1 , (b) 2 , (c) 4 .

The case where the in-plane shear strength is increased will be discussed next. Figure 14 shows the asymptotic crack-tip stress and strain fields for $n=3$. Figure 14(a) again gives the crack-tip stress and strain fields for isotropic materials. Figures 14(b) and 14(c) show the crack-tip stress and strain fields, $\bar{\sigma}_{i j}$ and $\bar{\varepsilon}_{i j}$, for $\sqrt{3} T_{0} / X_{0}=2$ and 4 (with $X_{0}=Y_{0}=Z_{0}$ ), respectively. Note that $\bar{\sigma}_{i j}=\left(\sqrt{3} T_{0} / X_{0}\right) \tilde{c}_{i j}$ and $\tilde{\varepsilon}_{i j}=\tilde{\varepsilon}_{i j} /\left(\sqrt{3} T_{0} / X_{0}\right)$. These figures show that when the in-plane shear strength is increased (or the tensile strength in the $x_{1}, x_{2}$, and $x_{3}$ directions is decreased), the angular functions $\tilde{\sigma}_{e}$ and $\tilde{\varepsilon}_{0 \theta}$ are intensified in front of the crack tip between $\theta=45^{\circ}$ and $\theta=-45^{\circ}$.

Figure 15 represents the asymptotic crack-tip stress and strain fields for $n=20$. Figure 15(a) shows the crack-tip stress and strain fields for isotropic materials. Figures 15(b) and 

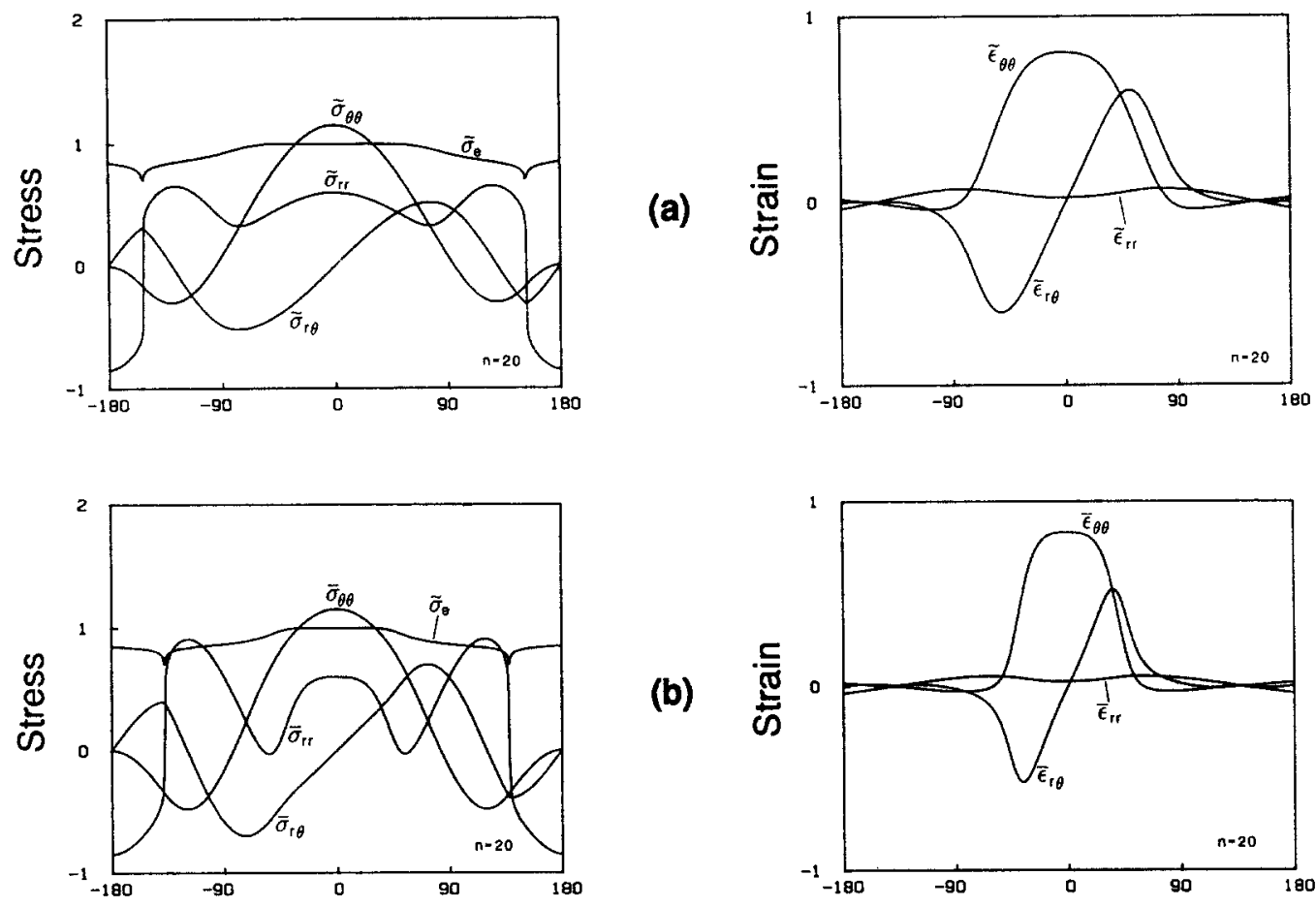

(a)

(b)
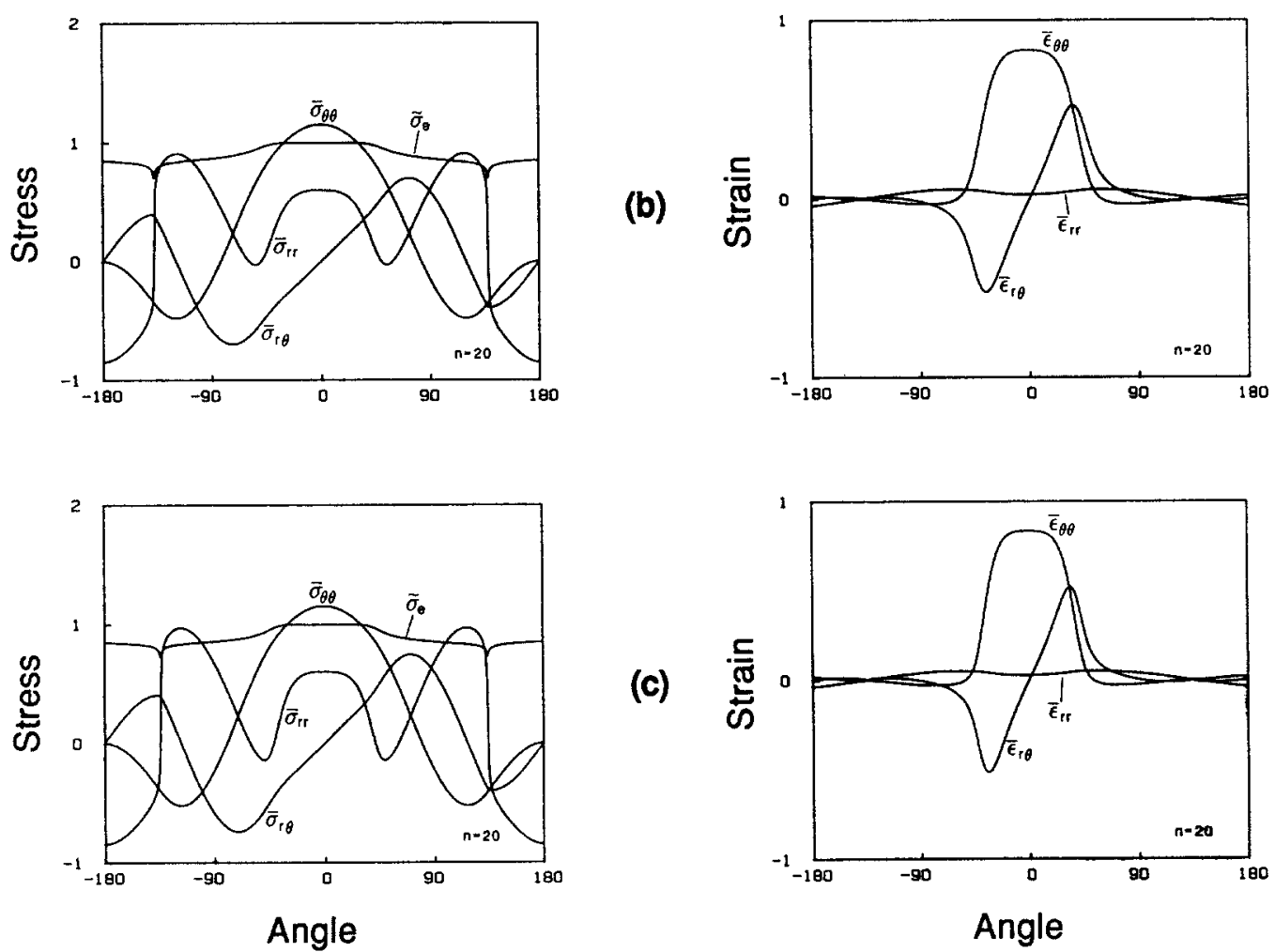

Fig. 15. Case 4: Angular distribution of the crack-tip stress and strain fields for $n=20, X_{0}=Y_{0}=Z_{0}$ and $\sqrt{3} T_{0} / X_{0}=$ (a) 1 , (b) 2 , (c) 4 .

15(c) give the crack-tip stress and strain fields for $\sqrt{3} T_{0} / X_{0}=2$ and 4 (with $X_{0}=Y_{0}=Z_{0}$ ), respectively. The trends of the crack-tip fields in Fig. 15 are similar to those in Fig. 14. An apparent discontinuity of the radial stress occurs where the angle $\theta$ decreases from $150^{\circ}$ to $130^{\circ}$.

Figures 16(a) and 16(b) show the effective stress contours for $n=3$ and $n=20$, respectively. For both $n=3$ and $n=20$, the effective stress contour grows ahead of the crack tip as the in-plane shear strength is increased (or the tensile strength in the $x_{1}, x_{2}$, and $x_{3}$ directions is decreased). Also, we can see that as $n$ increases, the area enclosed by the normalized effective stress contour increases. This characteristic is common to all four cases.

The perfectly plastic crack-tip solutions corresponding to the four cases that we discussed here are detailed in Pan [18]. A comparison of these perfectly plastic crack-tip stress solutions 


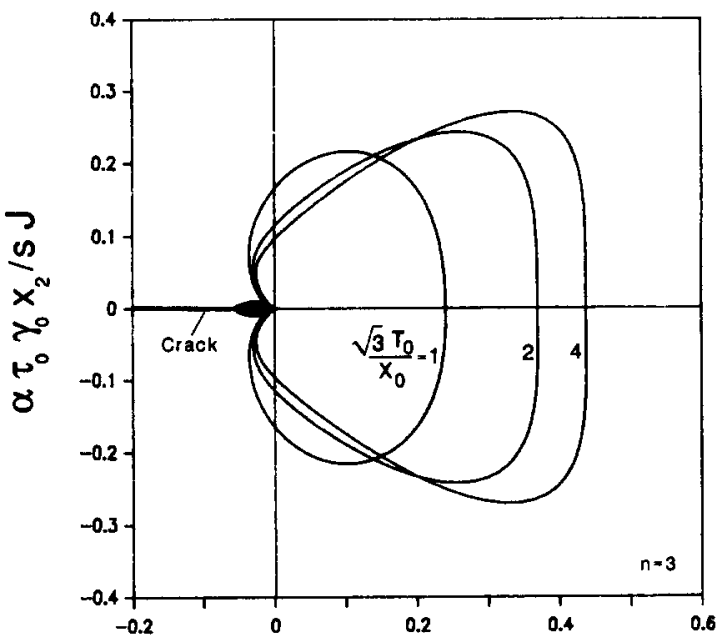

(a)

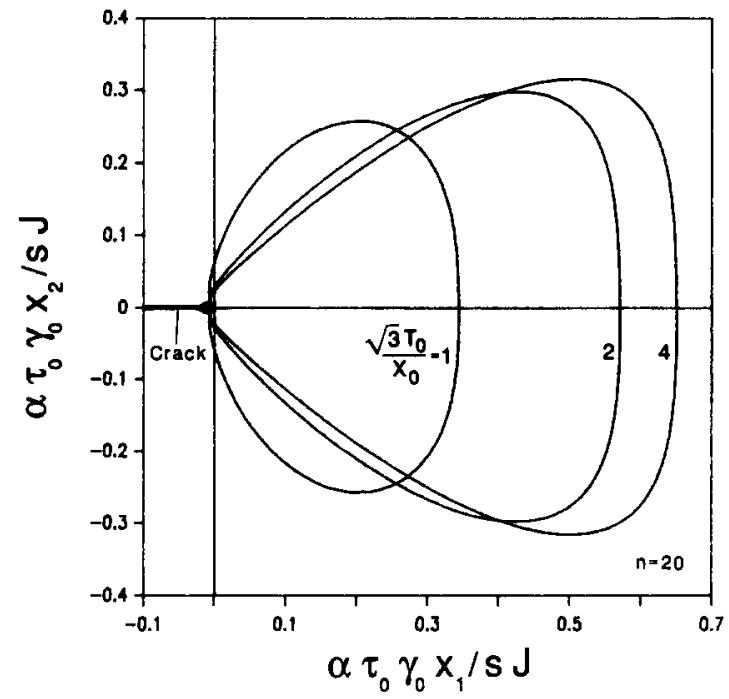

(b)

Fig. 16. Case 4: Effective stress contours plotted using the similarity coordinates for $X_{0}=Y_{0}=Z_{0}$ and $\sqrt{3} T_{0} / X_{0}=1,2$, and 4. (a) $n=3$, (b) $n=20$.

with the low-hardening solutions of $n=20$ in this study strongly suggests that the lowhardening limit of our asymptotic crack-tip solutions is indeed given by the perfectly plastic solutions presented in Pan [18].

\section{Discussions}

In this study, we only examine the two-dimensional crack-tip fields where the symmetry axes of orthotropy, $X_{1}, X_{2}$, and $X_{3}$, coincide with the present Cartesian coordinates, $x_{1}, x_{2}$, and $x_{3}$. If the symmetry axes of orthotropy do not coincide with the present Cartesian coordinates, remote tensile or remote shear stress will generally induce near-tip fields that are 
Table 1. The integration constant I, the normalized hoop stress, and the crack-tip opening displacement coefficient $D$ for Case 1

The integration constant $I$

\begin{tabular}{llll}
\hline$X_{0} / Y_{0}$ & $n=3$ & $n=10$ & $n=20$ \\
\hline 1 & 3.8549 & 2.9789 & 2.7416 \\
2 & 3.1513 & 2.6041 & 2.4460 \\
4 & 2.8026 & 2.5524 & 2.4398 \\
\hline
\end{tabular}

The normalized hoop stress

\begin{tabular}{lllll}
\hline$X_{0} / Y_{0}$ & $n=3$ & $n=10$ & $n=20$ & $n=\infty$ \\
\hline 1 & 1.0000 & 1.0000 & 1.0000 & 1.0000 \\
2 & 0.9371 & 0.9086 & 0.9015 & 0.8944 \\
4 & 0.9239 & 0.8869 & 0.8797 & 0.8729 \\
\hline
\end{tabular}

The crack-tip opening displacement coefficient $D$

\begin{tabular}{llll}
\hline$X_{0} / Y_{0}$ & $n=3$ & $n=10$ & $n=20$ \\
\hline 1 & 4.5289 & 3.3290 & 2.8469 \\
2 & 4.3492 & 3.2234 & 2.7816 \\
4 & 4.1138 & 3.1268 & 2.7005 \\
\hline
\end{tabular}

Table 2. The integration constant I, the normalized hoop stress, and the crack-tip opening displacement coefficient $D$ for Case 2

\begin{tabular}{|c|c|c|c|c|}
\hline \multicolumn{4}{|c|}{ The integration constant I } & \\
\hline$Y_{0} / X_{0}$ & $n=3$ & $n=10$ & $n=20$ & \\
\hline 1 & 3.8549 & 2.9789 & 2.7416 & \\
\hline 2 & 4.1405 & 3.7084 & 3.5079 & \\
\hline 4 & 3.8606 & 3.6234 & 3.4983 & \\
\hline \multicolumn{5}{|c|}{ The normalized hoop stress } \\
\hline$Y_{0} / X_{0}$ & $n=3$ & $n=10$ & $n=20$ & $n=\infty$ \\
\hline 1 & 1.0000 & 1.0000 & 1.0000 & 1.0000 \\
\hline 2 & 1.5761 & 1.7063 & 1.7445 & 1.7889 \\
\hline 4 & 2.6130 & 3.1277 & 3.2939 & 3.4915 \\
\hline
\end{tabular}

The crack-tip opening displacement coefficient $D$

\begin{tabular}{llll}
\hline$Y_{0} / X_{0}$ & $n=3$ & $n=10$ & $n=20$ \\
\hline 1 & 4.5289 & 3.3290 & 2.8469 \\
2 & 2.0108 & 1.4866 & 1.2681 \\
4 & 0.7451 & 0.4609 & 0.3742 \\
\hline
\end{tabular}

neither symmetric nor anti-symmetric. Furthermore, six material orthotropy constants $(F, G, H, L, M$, and $N$ ) will appear in the expressions of the near-tip HRR type fields (3.2).

The tensile hoop stress $\sigma_{\theta \theta}$ ahead of the tip of a stationary crack (at $\theta=0^{\circ}$ ) appears to play an important role in fracture initiation. We consider the hoop stress at a small fixed distance $r$ ahead of the crack tip within the zone dominated by the HRR field (3.2) at the same value of $J$. The values of the tensile hoop stress (normalized by the tensile hoop stress for isotropic materials) for the four cases are listed in Tables $1-4$ for a given set of $\alpha, \sigma_{0}$, and $\varepsilon_{0}$ (see a detailed discussion in [11]). For future reference, the values of the integration constant $I$ for the four cases are also listed in the tables. 
Table 3. The integration constant I, the normalized hoop stress, and the crack-tip opening displacement coefficient $D$ for Case 3

\begin{tabular}{|c|c|c|c|c|}
\hline$Z_{0} / X_{0}$ & $n=3$ & $n=10$ & $n=20$ & \\
\hline 1 & 3.8549 & 2.9789 & 2.7416 & \\
\hline 2 & 5.6920 & 4.8182 & 4.2730 & \\
\hline 4 & 5.6159 & 4.3662 & 3.9978 & \\
\hline \multicolumn{5}{|c|}{ The normalized hoop stress } \\
\hline$Z_{0} / X_{0}$ & $n=3$ & $n=10$ & $n=20$ & $n=\infty$ \\
\hline 1 & 1.0000 & 1.0000 & 1.0000 & 1.0000 \\
\hline 2 & 1.3062 & 1.5575 & 1.6414 & 1.7676 \\
\hline 4 & 1.4439 & 1.8235 & 1.9676 & 2.1871 \\
\hline
\end{tabular}

The crack-tip opening displacement coefficient $D$

\begin{tabular}{llll}
\hline$Z_{0} / X_{0}$ & $n=3$ & $n=10$ & $n=20$ \\
\hline 1 & 4.5289 & 3.3290 & 2.8469 \\
2 & 3.2482 & 1.9101 & 1.3718 \\
4 & 2.7941 & 1.5756 & 1.2242
\end{tabular}

Table 4. The integration constant I, the normalized hoop stress, and the crack-tip opening displacement coefficient $D$ for Case 4

The integration constant $I$

\begin{tabular}{llll}
\hline$\sqrt{3} T_{0} / X_{0}$ & $n=3$ & $n=10$ & $n=20$ \\
\hline 0.25 & 3.5659 & 3.3721 & 3.3161 \\
0.5 & 3.9526 & 3.5804 & 3.4593 \\
1 & 3.8549 & 2.9789 & 2.7416 \\
2 & 2.5186 & 1.8582 & 1.7032 \\
4 & 2.1187 & 1.6171 & 1.5005 \\
\hline
\end{tabular}

The normalized hoop stress

\begin{tabular}{lllll}
\hline$\sqrt{3} T_{0} / X_{0}$ & $n=3$ & $n=10$ & $n=20$ & $n=\infty$ \\
\hline 0.25 & 2.7980 & 3.4667 & 3.7060 & 4.0000 \\
0.5 & 1.6898 & 1.8609 & 1.9229 & 2.0000 \\
1 & 1.0000 & 1.0000 & 1.0000 & 1.0000 \\
2 & 0.5589 & 0.5231 & 0.5122 & 0.5000 \\
4 & 0.2918 & 0.2649 & 0.2577 & 0.2500 \\
\hline
\end{tabular}

The crack-tip opening displacement coefficient $D$

\begin{tabular}{llll}
\hline$\sqrt{3} T_{0} / X_{0}$ & $n=3$ & $n=10$ & $n=20$ \\
\hline 0.25 & 2.2305 & 1.1136 & 0.8519 \\
0.5 & 3.1724 & 2.0696 & 1.7139 \\
1 & 4.5289 & 3.3290 & 2.8469 \\
2 & 6.4359 & 4.4742 & 3.9771 \\
4 & 11.6546 & 8.6953 & 7.3074 \\
\hline
\end{tabular}

The crack-tip opening displacement also appears to be an important parameter for crack initiation and growth. Experimental observations and some computational works suggest that crack initiation and a limited amount of stable crack growth can be characterized by a critical value of the $J$ integral, $J_{c}$, and a $J$-resistance curve or by a critical value of the crack-tip opening displacement, $\delta_{c}$, and a $\delta$-resistance curve (see the work of Shih [23] for 
more references in this area). For power-law materials, the crack-tip opening displacement approaches zero as $r$ approaches zero. An effective crack-tip opening displacement was suggested by Rice in [21], in which the crack-tip opening displacement $\delta_{t}$ is defined as the opening distance between the intercept of two $45^{\circ}$-lines, drawn back from the tip, with the deformed crack profile. Then, based on the asymptotic crack-tip displacement solutions, $\tilde{u}_{r}$ and $\tilde{u}_{0}$, at $\theta=180^{\circ}$, the crack-tip opening displacement $\delta_{t}$ can be written in the form such that $\delta_{t}$ is proportional to the remote loading $J$ :

$$
\delta_{t}=d\left(J / \sigma_{0}\right)
$$

where $d$ is a constant for a given material and is expressed as

$$
d=D\left(\alpha \varepsilon_{0}\right)^{1 / n}
$$

where the coefficient $D$ is

$$
D=2\left(\tilde{u}_{r}+\tilde{u}_{0}\right) \tilde{u}_{0} / I
$$

where $\tilde{u}_{r}$ and $\tilde{u}_{\theta}$ are the crack-tip displacement solutions at $\theta=180^{\circ}$. The values of the coefficient $D$ for the four cases are listed in Tables 1-4.

A general trend between the normalized hoop stress ahead of the crack tip and the crack-tip opening displacement coefficient $D$ for cases 2,3 , and 4 where pronounced changes of crack-tip fields are observed is evident. For these cases, as the normalized hoop stress increases, the coefficient $D$ decreases. Let us examine the trend case by case.

For Case 1, the reinforcement is in the direction of the crack line. Except for the stress and strain along the crack line direction, the reinforcement has only a slight effect on the cracktip fields. This fact is reflected in Figs. 2-4 where the crack-tip stress and strain fields as well as the effective stress contours are plotted. The values of the normalized hoop stress and the crack-tip opening displacement coefficient D in Table 1 for Case 1 are slightly affected by the amount of reinforcement. Note that the values of the normalized hoop stress for $n=\infty$ presented in Tables 1-4 are based on the crack-tip solutions for the perfectly plastic materials presented in Pan [18]. Based on the trend of the perfectly plastic solutions, the slight decrease of the normalized hoop stress for power-law materials as the strength in the crack line direction increases can be accepted. However, the crack-tip opening displacement coefficient $\mathrm{D}$ decreases a bit as the strength in the crack line direction increases. This slight decrease of $D$ merely reflects the net effect of the reinforcement on the crack-tip opening displacement (see (5.1), (5.2), and (5.3)).

For Case 2, the reinforcement is in the direction perpendicular to the crack line. In this orientation, the reinforcement greatly influences the crack-tip stress and strain fields. This fact is reflected in Figs. 5-7 where the crack-tip stress and strain fields as well as the effective stress contours are plotted. In Table 2, as the strength in the $x_{2}$ direction is increased, the value of the normalized hoop stress increases and the value of the crack-tip opening displacement coefficient $D$ decreases.

For Case 3, the reinforcement is in the out-of-plane direction. As discussed earlier, the reinforcement restrains the deformation in the out-of-plane direction and promotes the plane-strain-like crack-tip deformation, which is reflected in Figs. 8-10. As indicated in Table 3, as the plane-strain-like constraint is increased due to the increase of the strength 
in the $x_{3}$ direction, the value of the normalized hoop stress in front of the crack tip increases and the value of the crack-tip opening displacement coefficient $D$ decreases. Note that for both Case 2 and Case 3, as the hardening exponent $n$ increases, the normalized hoop stress increases for a given ratio of $Y_{0} / X_{0}$ and $Z_{0} / X_{0}$, respectively.

For Case 4, the in-plane shear strength with respect to the $x_{1}$ and $x_{2}$ axes varies. As the in-plane shear strength is decreased (or increased), the value of the normalized hoop stress increases (or decreases) and the value of the crack-tip opening displacement coefficient $D$ decreases (or increases). Note that the decrease (or increase) of the in-plane shear strength is equivalent to the increase (or decrease) of the tensile strength in the $x_{1}, x_{2}$, and $x_{3}$ directions

- this follows from the definition of the effective stress adopted in this paper.

The hardening behavior of our orthotropic materials has been idealized by the power-law relation and the plastic orthotropy by Hill's quadratic yield function. These are rather restrictive assumptions. In particular, nonlinear tensile stress-strain curves of many engineering materials are not adequately described by a power-law relation. Our power-law constitutive relation for orthotropic materials does not accurately describe the real material behavior. The intent is to employ a simple constitutive model that retains certain essential aspects of plastic orthotropy in order to understand qualitatively the effects of plastic hardening and plastic orthotropy on the near-tip fields. These near-tip fields may provide the boundary condition for an idealized crack-tip model for some engineering materials including composite materials. The crack-tip model would allow the effect of detailed microstructure and plastic anisotropy on fracture toughness of these engineering materials to be investigated.

To describe the plastic anisotropic behavior, we used Hill's phenomenological quadratic yield function [1], which is smooth in the stress space. However, the yield surfaces based on the concept of crystalline slip for plastic deformation of single crystals and polycrystals well within the plastic range contain flat surfaces and vertices in the stress space. Rice [24] and Rice and Nikolic [25] have presented crack-tip fields for stationary cracks and growing cracks in single crystals under anti-plane shear conditions. Rice [26] has also studied crack-tip fields in single crystals under tensile loading conditions. Crack-tip stress and strain fields for orthotropic materials under plane-strain conditions have been presented by Pan and Shih [11] for power-law materials and by Pan [12] for perfectly plastic materials using the slip-line theory of Rice [27].

\section{Acknowledgement}

J.P. acknowledges the support of the National Science Foundation MSM-8613544 and the Rackham Faculty Grant of the University of Michigan. C.F.S. acknowledges the support of the Material Research Laboratory at Brown University, which is funded by the National Science Foundation through Grant DMR83-16893 and the US Department of Energy through Grant DE-AC02-80-ER10556.

\section{References}

1. R. Hill, Proceedings Royal Society London, Series A, 193 (1948) 281-291.

2. J.L. Bassani, International Journal of Mechanical Sciences 19 (1977) 651-660.

3. J.F.W. Bishop and R. Hill, Philosophical Magazine 7 (1951) 414-427. 
4. R. Hill, Mathematical Proceedings Cambridge Philosophical Society 85 (1979) 179-191.

5. J.W. Hutchinson, Journal of the Mechanics and Physics of Solids 16 (1968) 13-31.

6. J.W. Hutchinson, Journal of the Mechanics and Physics of Solids 16 (1968) 337-347.

7. J.R. Rice and G.F. Rosengren, Journal of the Mechanics and Physics of Solids 16 (1968) 1-12.

8. C.F. Shih, "Elastic-Plastic Analysis of Combined Mode Crack Problems," Ph.D. thesis, Harvard University (1973).

9. C.F. Shih, Fracture Analysis, ASTM STP 560, American Society for Testing and Materials (1974) $187-210$.

10. K. Hayashi, Journal of the Mechanics and Physics of Solids 27 (1979) 163-174.

11. J. Pan and C.F. Shih, the Mechanics of Materials 5 (1986) 299-316.

12. J. Pan, Journal of the Mechanics and Physics of Solids (1986) 617-635.

13. J.R. Rice, Transactions of the ASME, Journal of Applied Mechanics 35 (1968) 379-386.

14. J.W. Hutchinson, Transactions of the ASME. Journal of Applied Mechanics 50 (1983) 1042-1051.

15. Y.T. Yeow, D.H. Morris and H.F. Brinson, Experimental Mechanics 19 (1979) 1-8.

16. C.F. Shih, "Tables of Hutchinson-Rice-Rosengren Singular Field Quantities," MRL E-147, Material Research Laboratory, Brown University (1983).

17. J.R. Rice, in Mechanics of Solids: The R. Hill Anniversary Volume, H.G. Hopkins and M.J. Sewell (eds.), Pergamon Press, Oxford (1982) 539-562

18. J. Pan, "Plane-Stress Crack-Tip Stress Field for Perfectly Plastic Orthotropic Materials," submitted for publication.

19. N. Levy, P.V. Marcal, W.J. Ostergren and J.R. Rice, International Journal of Fracture Mechanics 7 (1971) $143-156$.

20. J.R. Rice and D.M. Tracey, in Numerical and Computer Methods in Structural Mechanics, S.J. Fenves et al., Academic Press, New York (1973).

21. D.M. Tracey, Transactions of the ASME, Journal of Engineering Materials and Technology 98 (1976) 146-151.

22. D.M. Parks, "Some Problems in Elastic-Plastic Finite Element Analysis of Cracks," Ph.D. thesis, Brown University, Providence, RI (1975).

23. C.F. Shih, Journal of the Mechanics and Physics of Solids 29 (1981) 305-326.

24. J.R. Rice, Mechanics of Materials 3 (1984) 55-80.

25. J.R. Rice and R. Nikolic, Journal of the Mechanics and Physics of Solids 33 (1985) 595-622.

26. J.R. Rice, "Tensile Crack Tip Fields in Elastic-Ideally Plastic Crystals," Report No. Mech-106, Division of Applied Sciences, Harvard University (July 1987) to be published.

27. J.R. Rice, Journal of the Mechanics and Physics of Solids 21 (1973) 63-74.

Résumé. On examine les champs de contraintes planes selon un mode I au voisinage de l'extrémité d'une fissure dans des matériaux orthotropes. L'orthotrope plastique est décrite par la fonction quadratique de plastification de Hill, et le comportement à l'écrouissage est donné par une généralisation adéquate d'une relation tensiondilatation de forme parabolique, sous traction mono-axiale. On a observé des modifications profondes dans l'aspect des variations angulaires des champs d'extrémité de fissure, selon le degré d'orthotropie plastique et l'intensité de l'écrouissage. Pour des matériaux très sujets ou peu sujets à l'écrouissage, et pour une large gamme d'orthotropies plastiques, on présente les formes et dimensions possibles des zones plastiques, telles qu'elles se déduisent des contours effectifs de contraintes. La forme de la zone plastique correspondant au cas particulier d'une orthotropie plastique s'accorde remarquablement bien à la zone de dilatation importante crée par une fissure d'orientation appropriée, dans une plaque de graphite-epoxy. 